\title{
Electroacupuncture improves repeated social defeat stress-elicited social avoidance and anxiety-like behaviors by reducing Lipocalin-2 in the hippocampus
}

\author{
Yi-Hung Chen ${ }^{1}$, Sheng-Yun Xie ${ }^{2}$, Chao-Wei Chen ${ }^{3}$ and Dah-Yuu Lü ${ }^{2,4^{*}}$ (i)
}

\begin{abstract}
Background: Post-traumatic stress disorder (PTSD) is a trauma-related disorder that is associated with pro-inflammatory activation and neurobiological impairments in the brain and leads to a series of affective-like behaviors. Electroacupuncture (EA) has been proposed as a clinically useful therapy for several brain diseases. However, the potential role of EA treatment in PTSD and its molecular and cellular mechanisms has rarely been investigated.

Methods: We used an established preclinical social defeat stress mouse model to study whether EA treatment modulates PTSD-like symptoms and understand its underlying mechanisms. To this end, male C57BL/6 mice were subjected to repeated social defeat stress (RSDS) for 6 consecutive days to induce symptoms of PTSD and treated with EA at Baihui (GV 20) and Dazhui (GV 14) acupoints.

Results: The stimulation of EA, but not needle insertion at Baihui (GV 20) and Dazhui (GV 14) acupoints effectively improved PTSD-like behaviors such as, social avoidance and anxiety-like behaviors. However, EA stimulation at the bilateral Tianzong (SI11) acupoints did not affect the PTSD-like behaviors obtained by RSDS. EA stimulation also markedly inhibited astrocyte activation in both the dorsal and ventral hippocampi of RSDS-treated mice. Using next-generation sequencing analysis, our results showed that EA stimulation attenuated RSDS-enhanced lipocalin 2 expression in the hippocampus. Importantly, using double-staining immunofluorescence, we observed that the increased lipocalin 2 expression in astrocytes by RSDS was also reduced by EA stimulation. In addition, intracerebroventricular injection of mouse recombinant lipocalin 2 protein in the lateral ventricles provoked social avoidance, anxiety-like behaviors, and the activation of astrocytes in the hippocampus. Interestingly, the overexpression of lipocalin 2 in the brain also altered the expression of stress-related genes, including monoamine oxidase A, monoamine oxidase B, mineralocorticoid receptor, and glucocorticoid receptor in the hippocampus.
\end{abstract}

Conclusions: This study suggests that the treatment of EA at Baihui (GV 20) and Dazhui (GV 14) acupoints improves RSDS-induced social avoidance, anxiety-like behaviors, astrocyte activation, and lipocalin 2 expression. Furthermore, our findings also indicate that lipocalin 2 expression in the brain may be an important biomarker for the development of PTSD-related symptoms.

*Correspondence: dahyuu@mail.cmu.edu.tw

2 Department of Pharmacology, School of Medicine, China Medical

University, Taichung, Taiwan

Full list of author information is available at the end of the article

(c) The Author(s) 2021. Open Access This article is licensed under a Creative Commons Attribution 4.0 International License, which permits use, sharing, adaptation, distribution and reproduction in any medium or format, as long as you give appropriate credit to the original author(s) and the source, provide a link to the Creative Commons licence, and indicate if changes were made. The images or other third party material in this article are included in the article's Creative Commons licence, unless indicated otherwise in a credit line to the material. If material is not included in the article's Creative Commons licence and your intended use is not permitted by statutory regulation or exceeds the permitted use, you will need to obtain permission directly from the copyright holder. To view a copy of this licence, visit http://creativecommons.org/licenses/by/4.0/. The Creative Commons Public Domain Dedication waiver (http://creativeco mmons.org/publicdomain/zero/1.0/) applies to the data made available in this article, unless otherwise stated in a credit line to the data. 
Keywords: Post-traumatic stress disorder, Electroacupuncture, Next-generation sequencing, Lipocalin-2, Intracerebroventricular injection, Repeated social defeat stress

\section{Background}

Post-traumatic stress disorder (PTSD), a common consequence of trauma, is a trauma and stressor-related disorder caused by exposure to severe traumatic events [1]. The clinical hallmarks of PTSD include avoidance of trauma reminders, re-experiencing a traumatic event, arousal, and hyperarousal symptoms. PTSD is an anxiety disorder that may persist for decades if not treated [2]. Repeated environmental stress puts patients at high risk for various psychiatric disorders that alter the morphology and activity of neurons in the central nervous system (CNS) [3] and induces maladaptive emotional and cognitive behaviors [4]. Social stress is a common environmental stress in human society that increases the risk of psychopathologies [5]. Repeated social defeat stress (RSDS) is a psychosocial stress animal model that induces symptoms of PTSD, such as emotional change and anxiety-like behavior [6, 7]. Importantly, experimental animals undergone repeatedly social defeat were exhibited social avoidance of the stressors [8]. RSDS was also reported to enhance vulnerability to subsequent adversity, which promotes anxiety recurrence of stress sensitization [9]. Our recent studies also demonstrated that mice subjected to RSDS led to symptoms of PTSD, including social avoidance and anxiety-like behaviors $[10$, $11]$.

Accumulating evidence has shown that psychological stress causes neuroinflammation and immune responses, which are important factors in the pathogenesis of psychiatric illnesses [12]. In clinical studies, the activation of the immune system contributes to chronic stress responses and is implicated in the development of mood disorders [13]. Rodents exposed to various environmental stressors increase circulating pro-inflammatory cytokines, which is consistent with clinical observations [14]. Social defeat has also been reported to affect proinflammatory immune processes, including variations in pro-inflammatory cytokines, such as interleukin (IL)-1 $\beta$, IL-6, and tumor necrosis factor (TNF)- $\alpha$ in the brain [15]. RSDS initiates the release of inflammatory Ly6Chigh monocytes and Ly-6c intermediate granulocytes into circulation, and these monocytes are characterized by glucocorticoid insensitivity [16]. Notably, RSDS also promotes the accumulation of peripheral monocytes in the brain and causes prolonged anxiety-like behavior for one more week [17]. In addition, using green fluorescent protein (GFP)-expressing bone marrow chimeras indicated that RSDS promoted the recruitment of peripheral macrophages in the hippocampus [18]. The previous finding also showed that RSDS increased the number of recruited monocytes that enhanced the inflammatory response and was required for the induction of anxietylike behavior [19].

There are at least two techniques used in acupuncture therapy: manual acupuncture (MA) and electroacupuncture (EA), a modified form of traditional manual acupuncture. A combines the therapeutic effects of transcutaneous electric nerve stimulation and MA, which can be standardized by frequency, current, waveform, and length [20]. Previous reports have shown that MA at Neiguan (PC6) improves acute restraint stress-induced anxiety in rats [21]. Clinically, low-frequency EA suppresses focal epilepsy and improves epilepsy-induced sleep disruption [22]. Importantly, increasing clinical studies have tested the efficacy of acupuncture on PTSD. A recent study reported the use of acupuncture intervention to reduce earthquake-related pain and psychological symptoms in affected individuals of earthquake-stricken areas of central Italy [23]. Surprisingly, a clinical trial observed that acupuncture exerts large treatment effects in patients with PTSD and maintains the reduction of symptoms for 3 months [24]. Further, a systemic review of clinical studies with meta-analysis found that acupuncture was effective for PTSD patients [25]. In addition, another systematic review and meta-analysis of acupuncture also suggests that acupuncture treatment in adults with PTSD is favored at post-intervention for depressive symptoms, anxiety symptoms, and sleep quality [26]. Furthermore, a variety of regulatory mechanisms have been proposed that act in improvement of EA stimulation in PTSD animal models [27]. EA reduces the anxiety symptoms by down regulating the hypothalamic-pituitary-adrenal (HPA) axis [28], upregulating hippocampal thioredoxin expression [29], increasing expression of BDNF, and TrkB signaling in amygdala [30], increasing expression of cannabinoid receptor in prefrontal cortex [31], and preventing hippocampal neurogenesis [32]. It has also been reported that EA treatment improves the sleep disturbance by regulating cytokine expression in hippocampus [33].

Chronic and repeated stress may facilitate the onset and exacerbate the symptoms of various psychiatric disorders, such as PTSD. We recently investigated the molecular mechanism of individual susceptibility to environmental stress, which led to unexpected discoveries in biomarkers after stress [11]. In this study, we used a 
preclinical mouse model of RSDS to elaborate the clinical symptoms of PTSD under social stress in the human society. Although many studies have reported the beneficial effects of acupuncture in many CNS disorders, the precise mechanism remains unknown. The present study elucidates the therapeutic effects of EA on RSDSinduced behavioral impairment. Our results also identified biomarkers of RSDS and developed EA treatments for stress-related mood disorders.

\section{Materials and methods}

\section{Animals, RSDS, and experimental design}

Eight-week-old male C57BL/6 mice were obtained from the National Laboratory Animal Center (Taipei, Taiwan). Under standard laboratory conditions $\left(21 \pm 2{ }^{\circ} \mathrm{C}\right.$, 12-h light/dark cycle, with food and water available ad libitum), the mice were housed in groups before and after the social defeat experiments and during behavioral testing. A total of 1325 -week-old CD-1 (ICR) male mice (National Laboratory Animal Center) were used as aggressors in the social defeat paradigm.

All animal experiments were performed in accordance with the Animal Care and Use Guidelines of China Medical University (IACUC Approval No. CMUIACUC-2019-139). The social defeat stress paradigm was performed according to a standard social defeat stress protocol [8] but with some modifications. The aggressor CD-1 mice were screened based on their aggressiveness to an experimental C57BL/ 6 mouse, as measured by the latency (must be $<60 \mathrm{~s}$ ) and the number of attacks (must attack in at least two consecutive sessions) during the observation period (180 s). RSDS was applied between 15:00 and 17:00 in a sound-attenuated room under dim light. The experimental C57BL/6 mice were subjected to a new CD-1 aggressor mouse (one CD-1 aggressor mouse attacked three C57BL/6 mice in each CD-1 mouse cage) for $2 \mathrm{~h}$ once daily for 6 consecutive days. After $2 \mathrm{~h}$ of physical contact, the experimental mice were separated and housed in their home cages for the next $22 \mathrm{~h}$. The social interaction, light-dark box, or elevated plus maze (EPM) tests were performed $24 \mathrm{~h}$ after the last defeat episode. For tissue collection after the behavioral test, the mice were euthanized, and the brain tissues were dissected and stored at $-80^{\circ} \mathrm{C}$.

\section{Next generation sequencing (NGS)}

In each group (Control, RSDS, and RSDS + EA group), 13 hippocampal tissues were pooled together as one group and sent for RNA sequencing. After the behavioral tests, the mice were sacrificed, and the hippocampal tissue was removed quickly and put in liquid nitrogen for quick freezing. The hippocampal tissue was stored at $-80{ }^{\circ} \mathrm{C}$ until tissue processing. Total RNA was extracted from the tissue using TRIzol reagent (Sigma-Aldrich, St. Louis, MO, USA). For RNA isolation, purified RNA was quantified at OD260 nm using an ND-1000 spectrophotometer (Nanodrop Technology, USA) and quantified using Bioanalyzer 2100 (Agilent Technology, USA) with an RNA 6000 LabChip kit (Agilent Technology, USA). The DNA libraries were used for cluster generation and sequencing using cBot Operation for HD V2.5 Reagent and HiSeq X Operation for HD v2.5 reagent v1.3 (Illumina). The sequence was determined using Illumina's sequencing-by-synthesis technology (Illumina, USA), and the sequencing data (FASTQ reads) were generated using Welgene Biotech's pipeline based on Illumina's base calling program bcl2fastq v2.20. Genes with $p$ values $\leq 0.05$, and $\geq$ twofold changes were considered to be significantly differentially expressed [34, 35]. Genes with low expression levels $(<0.3$ FPKM value) in these three groups were excluded. In addition, GeneMANIA5 was employed to generate protein-interaction network [36].

\section{Needle insertion and EA stimulation}

EA treatment in animal model was performed as described in our previous report [37]. Briefly, to evaluate the effects of needle insertion or EA, C57BL/6 mice were individually acclimated in rectangular observation boxes and anesthetized with $2 \%$ isoflurane. Under anesthesia via nose mask, the mice were arranged for needle insertion or EA stimulation. A pair of stainless-steel acupuncture needles were inserted into the murine equivalent of the acupoints, Dazhui (GV14) and Baihui (GV20), for 20 min once a day for 5 successive days. Meanwhile, the control mice were only anesthetized with isoflurane for $20 \mathrm{~min}$. The acupoint "Baihui (GV20, Governing vessel meridian 20)" is located at the vertex of the dorsal midline. The acupoint "Dazhui (GV14, Governing vessel meridian 14)," is located at the depression between the spinous processes of the seventh cervical and the first thoracic vertebrae on the dorsal midline. To accomplish this, acupuncture needles (Beijing Zhongyan Taihe Medical Instrument Co., Ltd.; $0.16 \times 7 \mathrm{~mm}$ ) were inserted to a depth of approximately $3 \mathrm{~mm}$, after which they were stimulated at a frequency of $2 \mathrm{~Hz}$ and an intensity of $1 \mathrm{~mA}$ for $20 \mathrm{~min}$ with a pulse width of $150 \mu \mathrm{s}$, using an EA Trio 300 stimulator (Ito, Japan). The acupoint Tianzong (SI11, small intestine meridian 11), is located in the region of the scapula, in the depression at the center of the subscapular fossa [38]. A sham EA was performed by bilateral insertion of a pair of stainless-steel acupuncture needles into the middle of each scapula, which is equivalent to the Tianzong (SI11). 


\section{Preparation of behavioral tests}

The social interaction, light-dark box, and EPM tests were performed $24 \mathrm{~h}$ after the last defeat episode. The mice were acclimatized to a separate room for at least $1 \mathrm{~h}$ prior to each test, and all experiments were performed under low light conditions. Between each test, the apparatus was cleaned with $75 \%$ ethanol. In addition, the light-dark box test was conducted at the first task, social interaction test was conducted at the second task, EPM test was conducted at the end, and each behavioral test was conducted within an interval of more than $2 \mathrm{~h}$.

\section{Behavioral tests I: social interaction test}

The social interaction test was performed as described in our previous studies $[10,11]$. Briefly, at the end of the social defeat paradigm, the experimental C57BL/6 mice were allowed to freely explore on an open field arena $(53 \mathrm{~cm} \times 53 \mathrm{~cm} \times 38 \mathrm{~cm})$ towards an unfamiliar target mice in a mesh cage $(10 \mathrm{~cm} \times 7 \mathrm{~cm} \times 38 \mathrm{~cm})$ and was measured in $5 \mathrm{~min}$. In the first 2.5-min session (target mice absent), the C57BL/6 mouse was introduced to the open field chamber with an empty mesh cage located at one end of the field. In the second 2.5-min session (target mice present), the mesh cage contained an unfamiliar CD-1 mice. The area $(24 \mathrm{~cm} \times 14 \mathrm{~cm})$ surrounding the mesh cage was defined as the interaction zone. The tract of the C57BL/6 mice, duration of the measurement period, and time spent in the interaction or corner zones $(8 \mathrm{~cm} \times 8 \mathrm{~cm})$ were measured using the Noldus EthoVision XT 12 behavioral tracking system (Noldus Information Technology, Wageningen, Netherlands). The social interaction ratio was obtained by dividing the time spent in the interaction zone when the target was present by the time spent in the interaction zone when the target was absent.

\section{Behavioral tests II: light-dark box test}

The light-dark box test uses a Plexiglas box $(45 \mathrm{~cm} \times 20 \mathrm{~cm} \times 29 \mathrm{~cm})$ divided into dark and light compartments separated by an open door (diameter, $10 \mathrm{~cm}$ ) located in the center of the partition at the floor level. At the start of the test, each mouse was placed in a dark chamber and the door was opened. Mice were allowed to freely explore the apparatus for $5 \mathrm{~min}$ and the Noldus EthoVision XT 12 behavioral tracking system (Noldus Information Technology, Wageningen, Netherlands). The time spent in the light and dark compartments was measured and analyzed.

\section{Behavioral tests III: EPM test}

The EPM test was performed according to our previous studies [10, 11]. Briefly, the EPM apparatus was made from white Plexiglas with a white floor and consisted of two opposite open arms $(30 \mathrm{~cm} \times 5 \mathrm{~cm} \times 39.5 \mathrm{~cm})$ and two enclosed arms $(30 \mathrm{~cm} \times 5 \mathrm{~cm} \times 39.5 \mathrm{~cm})$ surrounded by a $25.5 \mathrm{~cm}$-high white wall. These arms were connected by a central area $(5 \mathrm{~cm} \times 5 \mathrm{~cm})$, and the device was elevated $50 \mathrm{~cm}$ above the floor. At the start of the test, each mouse was placed at the end of the open arms facing the center of the maze. Mice were allowed to freely explore the apparatus for $5 \mathrm{~min}$ and the Noldus EthoVision XT 12 behavioral tracking system (Noldus Information Technology, Wageningen, Netherlands) was used to measure the time spent in the open and closed arms.

\section{Immunohistochemistry}

The dissected tissue was fixed in $10 \%$ neutral buffered formalin overnight and dehydrated in 30\% sucrose solution for 2 days at $4{ }^{\circ} \mathrm{C}$. The dissected tissue was placed on a tissue base mold and the entire tissue was covered with an FSC 22 frozen section compound (3801480; Leica Surgipath, Buffalo Grove, USA). The frozen tissues were sectioned to a thickness of $30 \mu \mathrm{m}$ using a cryotome, and placed on glass slides suitable for immunohistochemistry and immunofluorescence staining. Coronal slices on glass slides were quenched for endogenous peroxidases with $3 \%$ hydrogen peroxide, and blocked by incubation in $1 \%$ Triton X-100 and 5\% bovine serum albumin for $1 \mathrm{~h}$ The anti-glial fibrillary acidic protein (GFAP) primary antibody (Z0334, Agilent Dako, USA) was applied to the slides at a dilution of 1:50, followed by overnight incubation at $4{ }^{\circ} \mathrm{C}$. After several washes, the sections were incubated with the secondary antibody and HRP-conjugated streptavidin, and developed with diaminobenzene reagent for $5 \mathrm{~min}$. Bound antibodies were detected using an immunoperoxidase secondary detection system (Merck KGaA, Darmstadt, Germany), and the slices were then washed and mounted on glass slides with mounting medium (Leica Surgipath, Buffalo Grove, USA).

\section{Immunofluorescence staining}

In the double-labeling immunofluorescence assay, the brain slices were incubated with rabbit anti-lipocalin 2 antibody (bs-1373R, Bioss Antibodies, Woburn, Massachusetts, USA) and rat anti-GFAP (2.2B10, Invitrogen, Waltham, Massachusetts, USA) overnight at $4{ }^{\circ} \mathrm{C}$. After washing, the sections were incubated for $1 \mathrm{~h}$ with goat anti-rat IgG $(\mathrm{H}+\mathrm{L})$ Alexa Fluor 594 secondary antibodies (1:200; Invitrogen, Waltham, Massachusetts, USA) and goat anti-rabbit IgG Alexa Fluor 488 secondary antibodies (1:200; Invitrogen, Waltham, Massachusetts, USA) for $1 \mathrm{~h}$, and incubated for $3 \mathrm{~min}$ with DAPI $(0.5 \mathrm{mg} / \mathrm{mL}$ at a dilution of $1: 1000)$ counterstaining, and mounted on glass slides with mounting medium. The images were captured and analyzed by confocal microscopy (ANDOR Dragonfly high-speed confocal system, ANDOR, Dragonfly 200) with $20 \times$ and $100 \times$ objectives. 


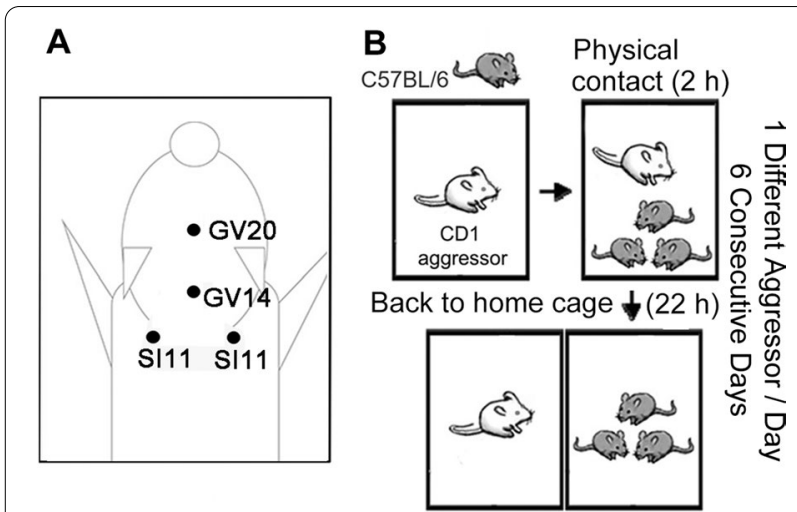

Fig. 1 Schematic diagrams of the experimental protocols. C57BL/6 mice were individually anesthetized via a nose mask. A pair of stainless-steel acupuncture needles were inserted into the murine equivalent of the acupoints: Dazhui (GV14) and Baihui (GV20), or bilateral Tianzong (SI11) (A). B The procedures of RSDS paradigm

\section{Reverse transcription and real-time polymerase chain reaction}

Real-time polymerase chain reaction (PCR) was performed to measure the expression levels of mineralocorticoid receptor (MR), glucocorticoid receptor (GR), monoamine oxidase (MAO)-A, MAO-B, lipocalin 2, and $\beta$-actin. Total RNA was isolated with TRIzol (SigmaAldrich, St. Louis, MO, USA), and the concentration of RNA was measured using a BioDrop spectrophotometer. The RT reactions were performed with $2 \mu \mathrm{g}$ of total RNA, which was reverse transcribed into cDNA. Target gene expression was detected using quantitative realtime PCR. PCR was performed using SYBR Green Master Mix (Applied Biosystems, Foster City, CA, USA) on a StepOne Plus Real-Time PCR System. The data were analyzed by comparing the cycle threshold $(\mathrm{Ct})$ values of the different groups using the method of $2^{-\Delta \Delta \mathrm{Ct}}$. The oligonucleotide primers were MR: 5- GAAGAGCCCCTC TGTTTGCAG -3 and 5- TCCTTGAGTGATGGGACT GTG -3; GR: 5- AGCTCCCCCTGGTAGAGAC -3 and 5GGTGAAGACGCAGAAACCTTG -3; MAO-A: 5- AAC GACTTGCTAAACTACAT -3 and 5- AGCAGAGAA GAGCCACAGAA -3; MAO-B: 5- GAAAACTGGTAC GTCTCACC -3 and 5- GAGCTGTTGCTGACAAGA TG -3; lipocalin 2: 5- CCCCATCTCTGCTCACTGTC -3 and 5- TTTTTCTGGACCGCATTG -3; $\beta$-actin: 5 - AGA GCTACGAGCTGCCTGAC -3 and 5- AGCACTGTG TTGGCGTACAG -3.

\section{Western blotting}

Tissue samples obtained from the hippocampus of the mice were homogenized in radioimmunoprecipitation lysis buffer containing protease and phosphatase inhibitor cocktails. Protein samples $(50 \mu \mathrm{g})$ were denatured in Laemmli buffer at $95{ }^{\circ} \mathrm{C}$ for $5 \mathrm{~min}$, separated by sodium dodecyl sulfate-polyacrylamide gel electrophoresis with a 15\% Tris-glycine gel electrophoresis, and then transferred on PVDF membranes (Millipore, Bedford, MA, USA). Membranes were blocked with non-fat dry milk (5\%) for $1 \mathrm{~h}$. and then incubated overnight at $4{ }^{\circ} \mathrm{C}$ with the following primary antibodies: anti-NGAL (sc-515876, Santa Cruz Biotechnology, Inc., Dallas, TX, USA) and GAPDH (G8795, Sigma-Aldrich, St. Louis, MO, USA). After washing with TBST buffer, the membranes were incubated with anti-mouse or anti-rabbit HRP-conjugated secondary antibodies. Protein bands were visualized using enhanced chemiluminescence and Kodak X-OMAT LS film (Eastman Kodak, Rochester, NY, USA). The data were quantified using an ImageJ software (National Institutes of Health, Bethesda, MD, USA).

\section{Intracerebroventricular injection}

The mice were anesthetized and positioned on a stereotaxic instrument, and the i.c.v. administration of $7 \mathrm{ng}$ mouse recombinant lipocalin 2 protein (1857-LC-050, Biotechne) $1 \mu \mathrm{g} / \mathrm{mL}$ in the lateral ventricles using a $10-\mu \mathrm{L}$ Hamilton syringe with a $26 \mathrm{~S}$-gauge needle (Hamilton, Reno, NV, USA) mounted to an automated microinjector (Holliston, MA, USA). The infusions were made at a rate of $0.5 \mu \mathrm{L} / \mathrm{min}$ for $10 \mathrm{~min}$. The i.c.v. injection was located at $-0.8 \mathrm{~mm}$ with respect to bregma, $1.5 \mathrm{~mm}$ to the right from the center, and $-2.0 \mathrm{~mm}$ in depth (Fig. 8A). At the end of the injection, the needle was left in place for an additional 10 min before being retracted by slowly withdrawing the syringe to prevent any reflux. The skull was then cleaned, and the incision was sutured.

\section{Statistical analysis}

The values were determined using GraphPad Prism 8 software (GraphPad Software, Inc., La Jolla, CA, USA). The results are presented as the mean \pm standard error of mean. Statistical analysis between two samples was performed using the Student's t-test. For multiple comparisons, one-way analysis of variance (ANOVA) was performed, followed by the Bonferroni or Tukey tests. In all cases, a $p$-value $<0.05$, was considered to be statistically significant. The $p$-values are indicated in the "Results" and "Figure legends" sections. No pretest was used to determine the sample size. 


\section{(See figure on next page.)}

Fig. 2 Electroacupuncture stimulation at the Baihui (GV 20) and Dazhui (GV 14) acupoints improves RSDS-evoked social avoidance behavior. A Representative heat maps for the social activities of each group. After RSDS, the experimental mice were introduced to the social apparatus (Interaction zone: grey area; corner zones: green areas). The time spent in the interaction zone was determined when the target was present (B) or absent (C). D The social interaction ratio was calculated by dividing the time spent in the interaction zone while the target was present by the time spent in interaction zone while the target was absent. The time spent in corners were determined by the interaction zone with target $(\mathbf{E})$ or without target $(\mathbf{F})$. G The velocity traveled with the target present during the test. Quantitative data are presented as the mean \pm SEM (control: $n=15 ;$ RSDS: $n=15 ;$ RSDS/Sham: $n=14 ;$ RSDS/EA $n=15$ ). One-way ANOVA with a post-hoc Tukey test was used to examine the significance of the mean. ${ }^{*} p<0.05$ vs. the control group. ${ }^{* *} p<0.01$ vs. the control group. \# $p<0.05$ vs. the RSDS group. \#\# $p<0.01$ vs. the RSDS group. NS: not significant

\section{Results}

EA stimulation at the Baihui (GV 20) and Dazhui (GV 14) acupoints improves RSDS-induced social avoidance C57BL/6 mice were individually anesthetized via a nose mask, and treated with EA at the following acupoints: Dazhui (GV14) and Baihui (GV20), or bilateral Tianzong (SI11) (Fig. 1A). The RSDS paradigm was shown in Fig. 1B. First, to measure the effect of EA treatment in RSDS-treated mice, we determined the behaviors of EA-stimulated mice at the Baihui and Dazhui acupoints (GV20 and GV14) or by needle insertion (sham group). Figure $2 \mathrm{~A}$ shows the representative heat maps of the social interaction analysis following RSDS. RSDS mice treated with sham stimulation, like the RSDS group, spent less time interacting when a target was present compared with the control group. RSDS mice treated with EA stimulation spent more time interacting when a target was present, compared with the RSDS group. In addition, RSDS mice treated with EA spent a similar amount of time in the interaction zone with the target present, similar to the control group (Fig. 2B). There was no significant difference in the time spent in the interaction zone between the sham and RSDS groups (Fig. 2B, RSDS vs. control, $p<0.01$; RSDS + sham vs. RSDS, NS; RSDS + EA vs. RSDS, $p<0.01$; one-way ANOVA). As shown in Fig. 2C, there was no significant difference in the time spent in the interaction zone with the target mice absent in each group. Similarly, the analysis of the social interaction ratio of the RSDS mice was improved by EA treatment but not by sham treatment (Fig. 2D; RSDS vs. control, $p<0.05$; RSDS + sham vs. RSDS, NS; RSDS + EA vs. RSDS, $p<0.01$; one-way ANOVA). In contrast, the time spent in the corner zones of RSDS was also significantly reduced by EA stimulation but not by sham treatment (Fig. 2E; RSDS vs. control, $p<0.05 ; \mathrm{RSDS}+$ sham vs. RSDS, NS; RSDS + EA vs. RSDS, $p<0.05$; one-way ANOVA). There was no significant difference in the time spent in the corner zone with the target mice absent between the groups (Fig. 2F). In addition, movement velocity was not significantly different between the groups (Fig. 2G).
Collectively, these data indicate that EA stimulation at the Baihui and Dazhui acupoints improves RSDSinduced social avoidance and exerts potential therapeutic effects compared to needle insertion.

\section{EA stimulation at the Baihui (GV 20) and Dazhui (GV 14) acupoints improves RSDS-elicited anxiety-like behaviors} The affective-like activities of RSDS mice treated with EA were determined. Light and dark (L/D) box task has been widely used to examine anxious behavior in rodent models [39]. Figure 3A shows the representative heat maps of the light compartment in the L/D box following RSDS. The RSDS group spent less time traveling in the light compartment than the control group. The time spent traveling in the light compartment was significantly increased in the group of RSDS mice treated with EA, compared with the sham group (Fig. 3B). In addition, both the RSDS and RSDS groups with sham treatment spent a similar amount of frequency traveling in the light compartment (Fig. 3B). The mice in the RSDS group with EA treatment traveled more frequently in the light compartment than those in the RSDS group. (RSDS vs. control, $p<0.001$; RSDS + sham vs. RSDS, NS; RSDS + EA vs. RSDS, $p<0.001$; one-way ANOVA). The latency to the first entry into the light compartment of the RSDS group was markedly increased compared to that of the control group (Fig. 3C). Surprisingly, the latency to the first entry into the light compartment was also decreased by EA stimulation but not by sham treatment (Fig. 3C; RSDS vs. control, $p<0.0001$; RSDS + sham vs. RSDS, NS; RSDS + EA vs. RSDS, $p<0.001$; one-way ANOVA). Moreover, the cumulative time spent in the light compartment of the group of RSDS mice treated with EA was significantly increased compared to that in the RSDS group (Fig. 3D; RSDS vs. control, $p<0.0001$; RSDS + sham vs. RSDS, NS; RSDS + EA vs. RSDS, $p<0.01$; one-way ANOVA). In addition, there was no significant difference in the movement velocity between the groups (Fig. 3E).

We also used an EPM task to measure anxiety-like behaviors [40]. As shown in Fig. 4A, the trace recorded of RSDS mice exhibited a preference remaining in closed 

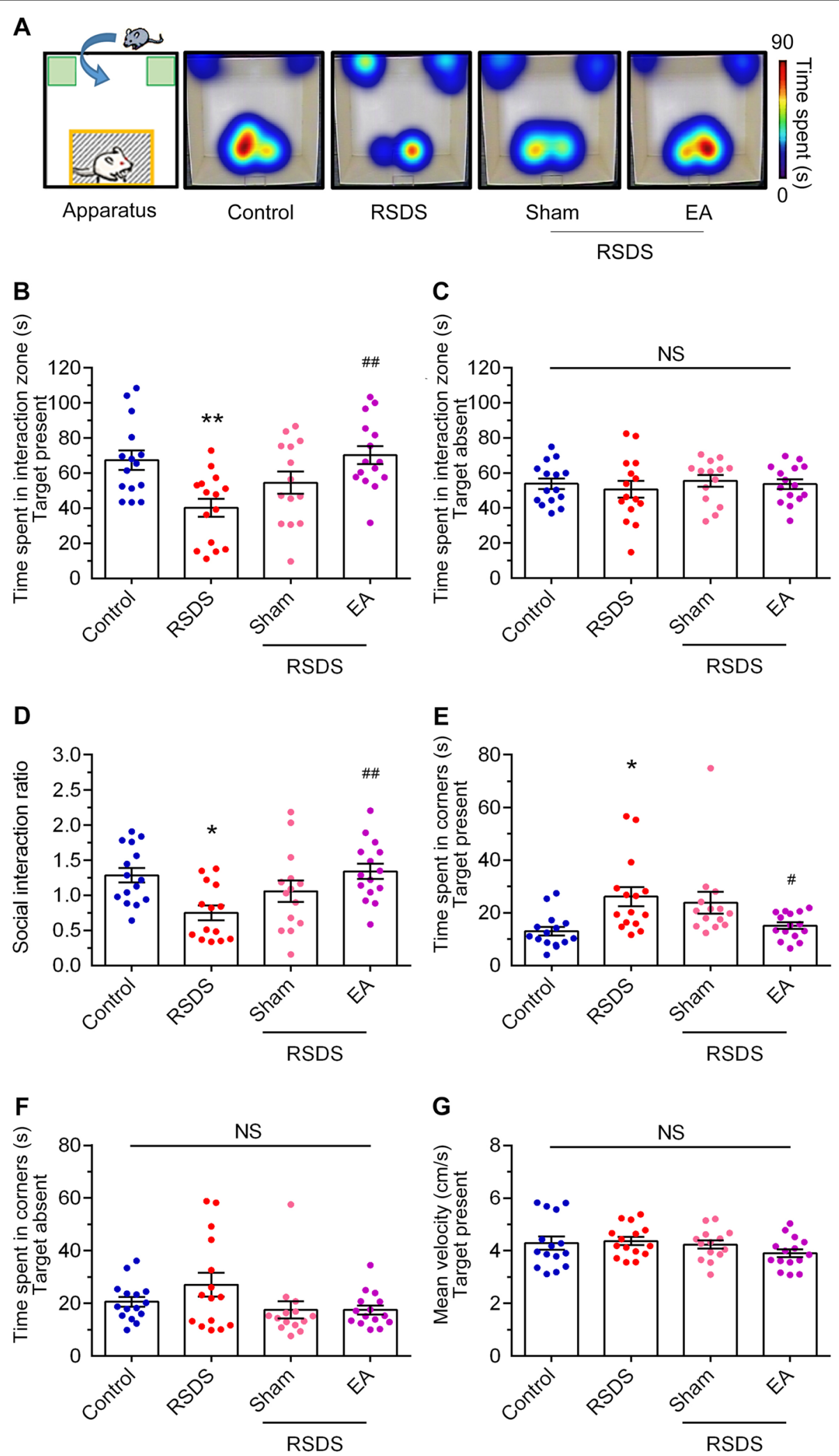

Fig. 2 (See legend on previous page.)

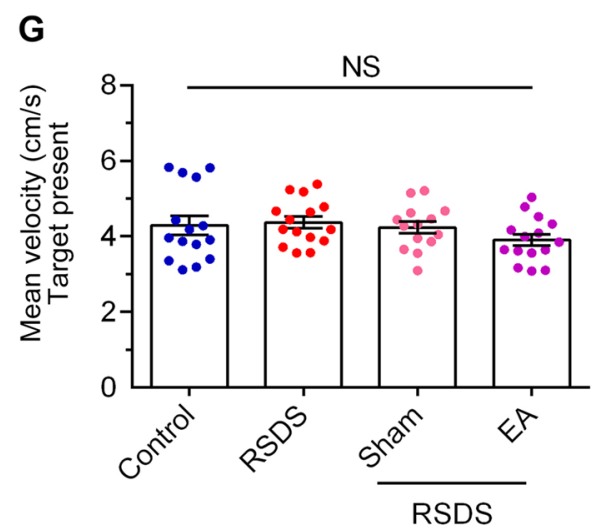


A

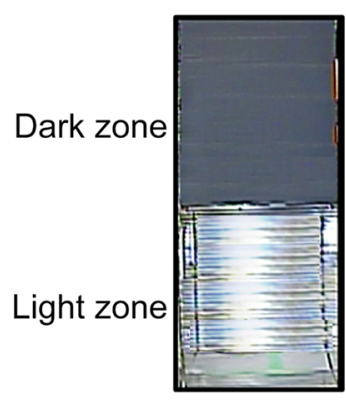

Apparatus

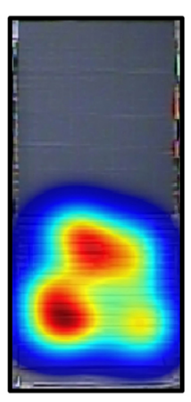

Control

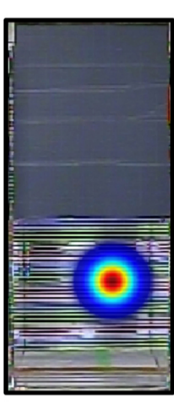

RSDS

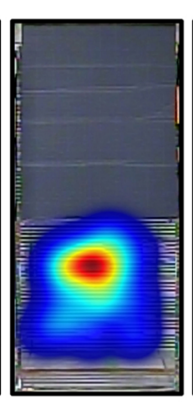

Sham

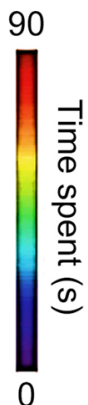

EA

RSDS

B

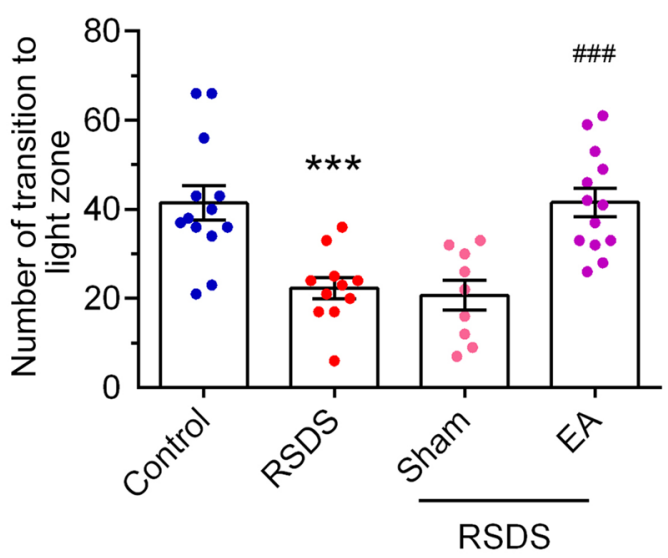

C
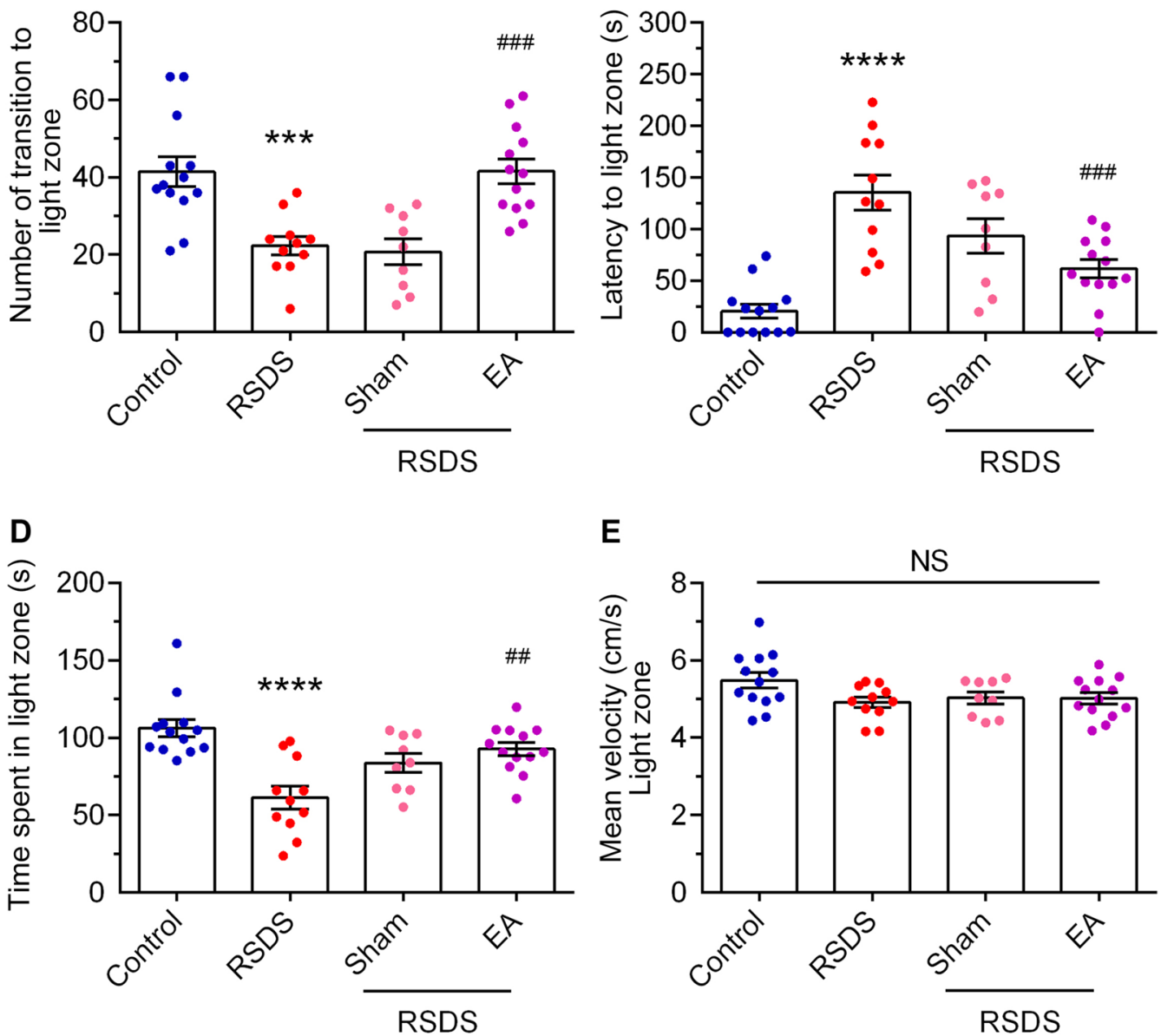

E

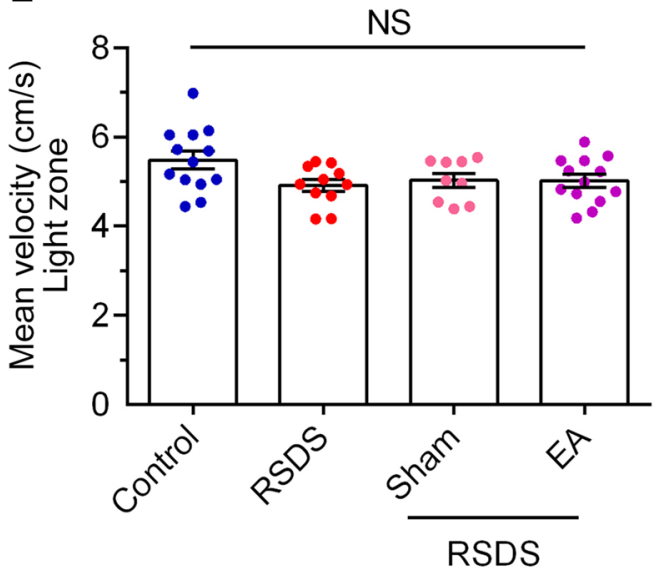

Fig. 3 EA stimulation at the Baihui (GV 20) and Dazhui (GV 14) acupoints improves RSDS-evoked anxiety-like behaviors in the light-dark box task. A Representative heat maps for the activities of each group during the light-dark test. The frequency spent in the light compartment (B), the latency to the first entry into light compartment $(\mathbf{C})$, or the cumulative time in the light compartment (D) were determined. The velocity traveled in the light compartment was measured in (E). Quantitative data are presented as the mean \pm SEM (control: $n=13 ;$ RSDS: $n=11 ; R S D S /$ Sham: $n=9 ;$ RSDS/EA $n=13$ ). One-way ANOVA with a post-hoc Tukey test was used to examine the significance of the mean. ${ }^{* * *} p<0.001$ vs. the control group. ${ }^{* * *} p<0.0001$ vs. the control group. \#\# $p<0.01$ vs. the RSDS group. \#\#\# $p<0.001$ vs. the RSDS group. ANOVA: analysis of variance, EA: electroacupuncture, NS: not significant, RSDS: repeated social defeat stress, SEM: standard error of mean 
A

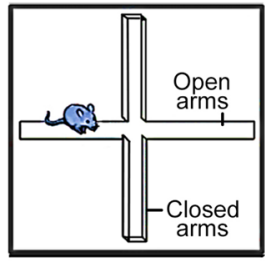

Apparatus

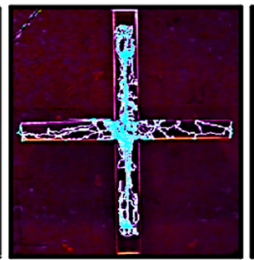

Control

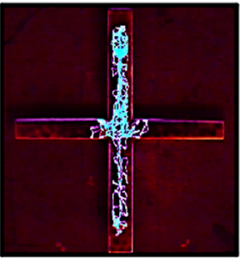

RSDS

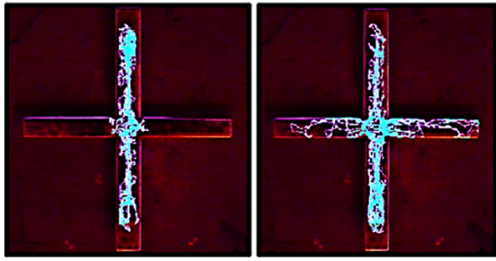

EA

Sham

RSDS
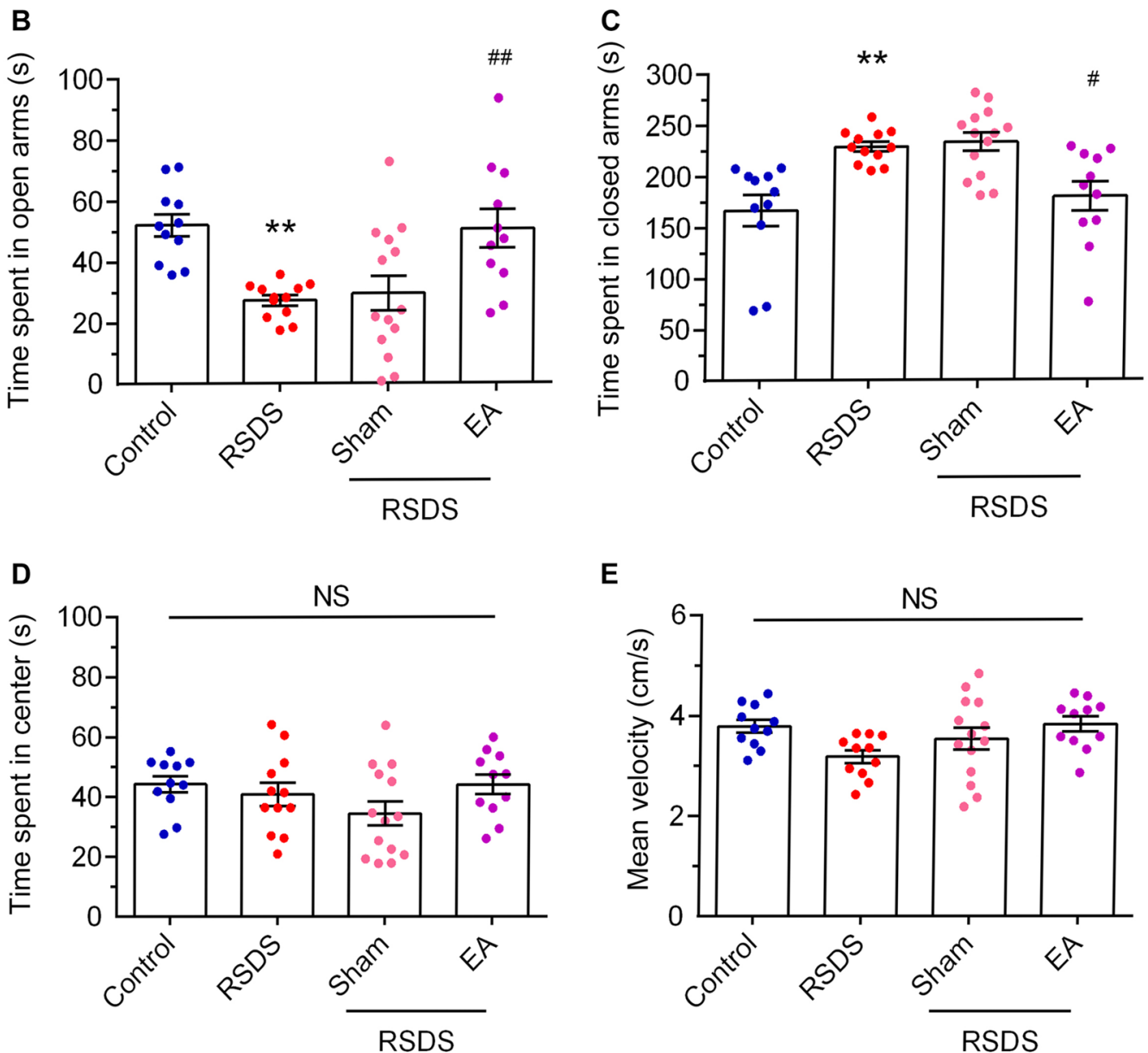

E

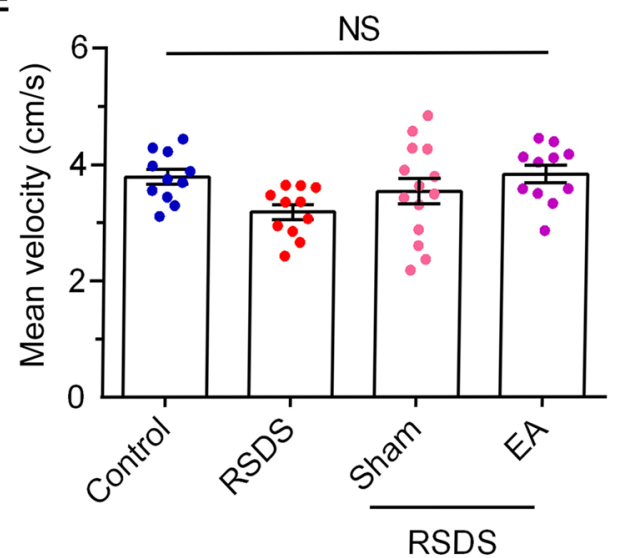

Fig. 4 EA stimulation at the Baihui (GV 20) and Dazhui (GV 14) acupoints improves RSDS-evoked anxiety-like behaviors in EPM task.

(A) Representative traces for the activity on EPM apparatus for each group. The time spent in open arms (B), closed arms (C), or center (D) of the EPM apparatus were determined. The velocity traveled on the EPM apparatus was measured in (E). Quantitative data are presented as the mean \pm SEM (control: $n=11$; RSDS: $n=12 ;$ RSDS/Sham: $n=14 ;$ RSDS/EA $n=11$ ). One-way ANOVA with a post-hoc Tukey test was used to examine the significance of the mean. ${ }^{* *} p<0.01$, the RSDS group vs. the control group. \# $p<0.05$, the EA group vs. the RSDS group. \#\# $p<0.01$, the EA group vs. the RSDS group. ANOVA: analysis of variance, EA: electroacupuncture, EPM: elevated plus maze, NS: not significant, RSDS: repeated social defeat stress, SEM: standard error of mean

arms compared to open arms during the EPM test. The time spent in the open arms was longer in the RSDStreated with EA group than in the RSDS group (Fig. 4B; RSDS vs. control, $p<0.01$; RSDS + sham vs. RSDS, NS;
RSDS + EA vs. RSDS, $p<0.01$; one-way ANOVA). As shown in Fig. 4C, the RSDS group treated with EA reduced the time spent in the closed arms compared to the RSDS group with sham treatment (RSDS vs. control, 


\section{(See figure on next page.)}

Fig. 5 EA stimulation at the Baihui (GV 20) and Dazhui (GV 14) acupoints down-regulates astrocyte activation in RSDS-treated mice. After the behavioral tests, the mice were sacrificed, and brain coronal slices were fixed for immunohistochemistry with an anti-GFAP antibody. Representative images were taken from dorsal (A) or ventral (B) hippocampal CA3 areas, and GFAP immunoreactivity was quantified as the percentage of GFAP-positive cells from the mice of the control group, RSDS, RSDS with sham treatment, or RSDS with EA treatment. Quantitative results of the dorsal (C) and ventral (D) hippocampi are presented as the mean \pm SEM. (control: $n=16$; RSDS: 17; RSDS/Sham: $n=17$; RSDS/EA $n=16$ ) One-way ANOVA with a post-hoc Bonferroni test was used to examine the significance of the mean. ${ }^{*} p<0.05$ vs. the control group. ${ }^{* * *} p<0.001$ vs. the control group. \# $p<0.05$ vs. the RSDS group. \#\# $p<0.01$ vs. the RSDS group. ANOVA: analysis of variance, EA: electroacupuncture, GFAP: glial fibrillary acidic protein, RSDS: repeated social defeat stress, SEM: standard error of mean

$p<0.01 ;$ RSDS + sham vs. RSDS, NS; RSDS + EA vs. RSDS, $p<0.05$; one-way ANOVA). There was no significant difference in the time spent in the center of the EPM apparatus between the groups (Fig. 4D). In addition, the velocity traveled in the EPM apparatus was not significantly different between the groups (Fig. 4E). Collectively, these results indicate that EA stimulation at the Baihui and Dazhui acupoints prevents RSDS-induced anxiety-like behaviors.

\section{Effects of EA stimulation at the bilateral Tianzong (SI11) acupoints}

To assess the effect of EA stimulation at the Tianzong acupoints in RSDS-treated mice, we performed EA stimulation at the bilateral Tianzong (SI11) acupoints (EA SI11 group). In the social interaction task, the time spent in the interaction zone with the target present was significantly increased in the group treated with RSDS and EA at Baihui (GV 20) and Dazhui (GV 14) acupoints (Additional file 1: Fig. S1A). However, there was no significant difference between the EA stimulation at the bilateral Tianzong (SI11) acupoints and the RSDS group (Additional file 1: Fig. S1A; RSDS vs. control, $p<0.001$; RSDS + EA vs. RSDS, $p<0.01$; RSDS + EA SI11 vs. RSDS, NS; one-way ANOVA). In addition, there was no significant difference in the time spent in the interaction zone with the target absent between the groups (Additional file 1: Fig. S1B). Similarly, the group of RSDS-treated with EA at the Baihui (GV 20) and Dazhui (GV 14) acupoints showed a significantly increased social interaction ratio compared with the control group. However, stimulation with EA bilateral Tianzong (SI11) acupoints did not have improved effects in RSDS-treated mice (Additional file 1: Fig. S1C; RSDS vs. control, $p<0.01$; RSDS + EA vs. RSDS, $p<0.01$; RSDS + EA SI11 vs. RSDS, NS; one-way ANOVA). In parallel, EA stimulation at the Baihui (GV 20) and Dazhui (GV 14) acupoints improved the time spent in the corner zone with the target present compared to the RSDS group, but this was not observed at the bilateral Tianzong (SI11) acupoints (Additional file 1: Fig. S1D; RSDS vs. control, $p<0.01 ;$ RSDS + EA vs.
RSDS, $p<0.01$; RSDS + EA SI11 vs. RSDS, NS; one-way ANOVA). There was no significant difference in the time spent in the corner zone with the target absent between the groups (Additional file 1: Fig. S1E).

In the L/D box task, the group of RSDS mice showed less frequency traveling in the light compartment than the control group (Additional file 2: Fig. S2A). Importantly, EA treatment at Baihui (GV 20) and Dazhui (GV 14) increased the frequency of travel spent in the light compartment, but not in EA bilateral Tianzong (SI11) stimulation (Additional file 2: Fig. S2A; RSDS vs. control, $p<0.01$; RSDS + EA vs. RSDS, $p<0.05$; RSDS + SI11 vs. RSDS, NS; one-way ANOVA). In addition, RSDSinduced an increased latency to the first entry into the light compartment which was reduced by EA treatment at the Baihui (GV 20) and Dazhui (GV 14) acupoints but not at the bilateral Tianzong (SI11) acupoints. (Additional file 2: Fig. S2B; RSDS vs. control, $p<0.001$; RSDS + EA vs. RSDS, $p<0.05$; RSDS + EA SI11 vs. RSDS, NS; one-way ANOVA). Moreover, the decreased cumulative time spent in the light compartment evoked by RSDS was also significantly increased by EA treatment at Baihui (GV 20) and Dazhui (GV 14) but not bilateral Tianzong (SI11) acupoints (Additional file 2: Fig. S2C; RSDS vs. control, $p<0.001$; RSDS + EA vs. RSDS, $p<0.05$; RSDS + EA vs. RSDS, NS; one-way ANOVA).

Similarly, EA treatment at the Baihui (GV 20) and Dazhui (GV 14) acupoints increased the time spent in open arms in the EPM task; however, this was not observed in EA stimulation at the bilateral Tianzong (SI11) acupoints (Additional file 3: Fig. S3A; RSDS vs. control, $p<0.05 ; \operatorname{RSDS}+\mathrm{EA}$ vs. RSDS, $p<0.05$; RSDS + SI11 vs. RSDS, NS; one-way ANOVA). In addition, there was no significant difference in the time spent in the center of the EPM task between each group (Additional file 3: Fig. S3B) Collectively, these findings indicate the specific acupoints of preventive effects on EA stimulation at the Baihui (GV 20) and Dazhui (GV 14) acupoints but not in the bilateral Tianzong (SI11) acupoints in RSDS-evoked social avoidance and anxiety-like behaviors. 
A

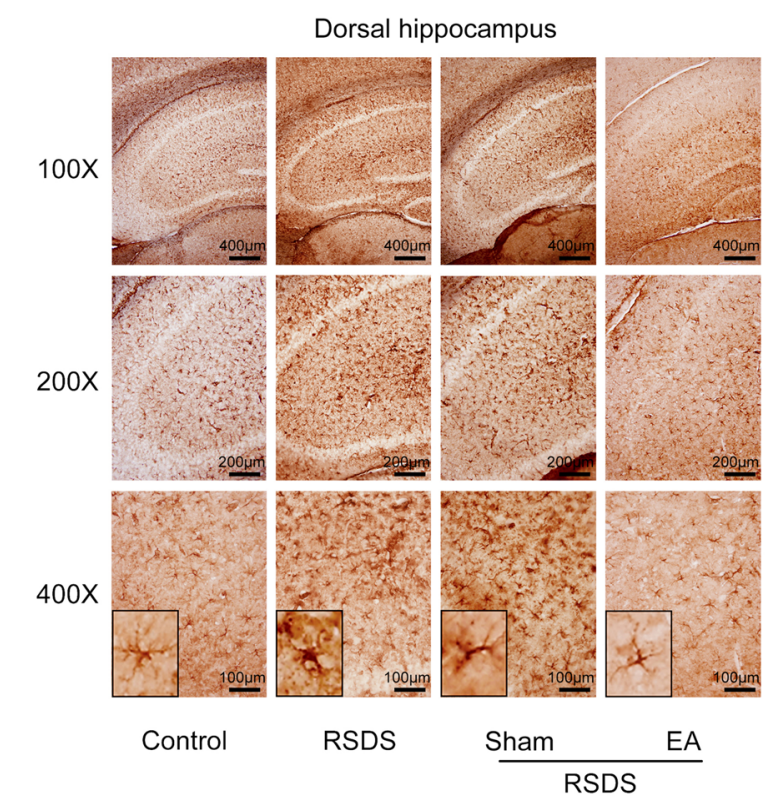

B

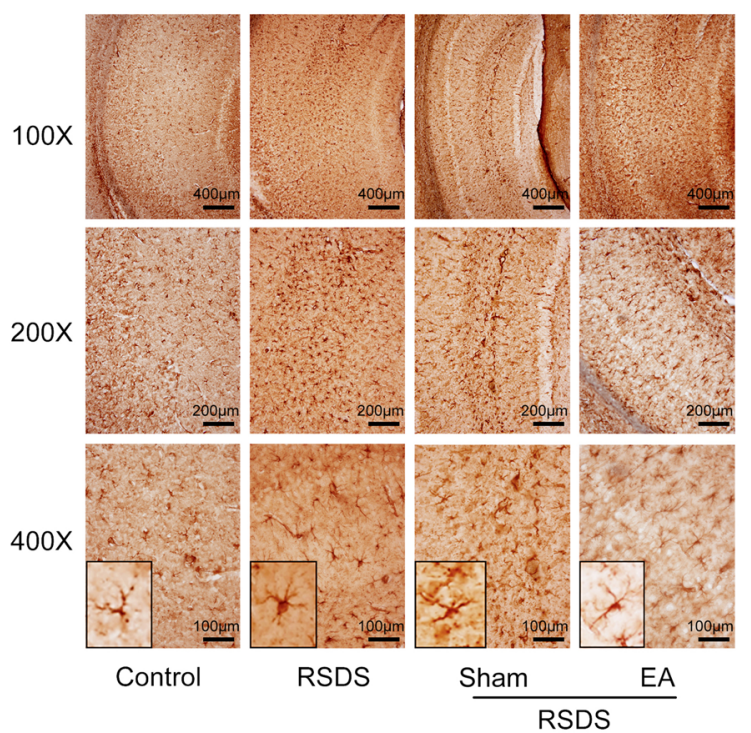

C

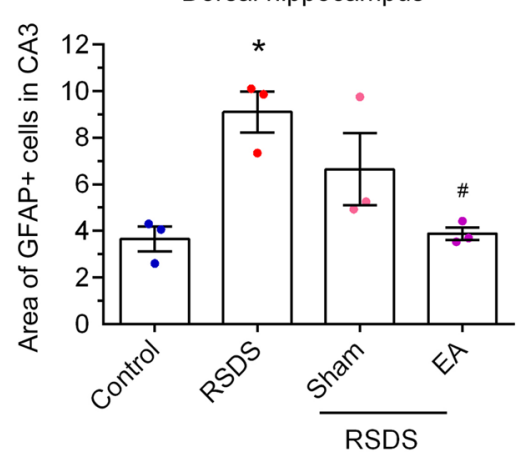

D Ventral hippocampus

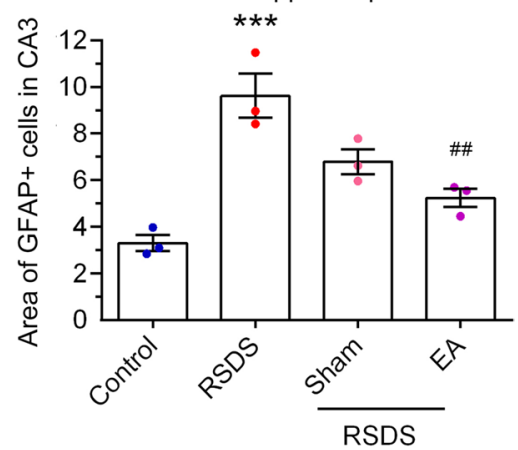

Fig. 5 (See legend on previous page.) 
(See figure on next page.)

Fig. 6 EA stimulation at the Baihui (GV 20) and Dazhui (GV 14) acupoints down-regulates the expression of lipocalin 2 in the hippocampus elicited by RSDS. Hippocampal tissues were collected after the behavior test. A Heat map representing the change in gene expression in the control, RSDS, and RSDS-treated with EA groups. Induced gene sets are shown in red, and repressed gene sets are shown in green. Differentially downregulated (blue spots, left panel) and upregulated (red spots, right panel) gene expression profiling with $p<0.05$, in the control versus RSDS groups (B) and RSDS versus RSDS + EA groups (C) were identified using the volcano plot. Genes with - $\log 10$ ( $p$-value) $>1.3$, and fold change $>2$ were selected for further analyses. mRNA expression (D) and protein expression (E) of lipocalin 2 were determined by real-time PCR and western blotting, respectively. Quantitative results are presented as the mean \pm SEM. One-way ANOVA with a post-hoc Tukey test was used to examine the significance of the mean. ${ }^{* *} p<0.001$ vs. the control group. \#\#\# $p<0.001$ vs. the RSDS group. ANOVA: analysis of variance, EA: electroacupuncture, PCR: polymerase chain reaction, RSDS: repeated social defeat stress, SEM: standard error of mean

\section{EA stimulation at the Baihui (GV 20) and Dazhui (GV 14) acupoints inhibits the astrocyte activation in hippocampus in RSDS-treated mice}

As shown in Fig. 5A, there were prominent astrogliosis throughout the dorsal hippocampus CA3 region in RSDS-stimulated mice compared with the control groups. In addition, the morphological characteristics of astrocyte activation in the ventral hippocampus were also observed, including a hypertrophic appearance with thick, densely labeled processes, and large cell bodies (Fig. 5B). However, astrocyte activation in the RSDS mice brain were attenuated by EA stimulation, compared to the sham treatment. As shown in Fig. $5 \mathrm{C}$ and D, the quantitative results of GFAP immunoreactivity were significantly increased in both the dorsal (RSDS vs. control, $p<0.05$; one-way ANOVA) and ventral (RSDS vs. control, $p<0.001$; one-way ANOVA) hippocampus in the RSDS group compared to the control group. Importantly, GFAP immunoreactivity was significantly decreased by EA treatment in the dorsal (Fig. 5C, RSDS + EA vs. RSDS, $p<0.05$; one-way ANOVA) and ventral (Fig. 5D, RSDS + EA vs. RSDS, $p<0.01$; one-way ANOVA) hippocampal CA3 region compared to the control group. In addition, the sham treatment had no effect on GFAP immunoreactivity (Fig. 5C and D). Collectively, these data indicate that EA stimulation at the Baihui (GV 20) and Dazhui (GV 14) acupoints prevents RSDS-induced astrocyte activation in the hippocampus.

\section{EA stimulation at the Baihui (GV 20) and Dazhui (GV 14) acupoints down-regulates lipocalin 2 expression in the hippocampal astrocytes in RSDS-treated mice}

To further identify the mechanism of EA treatment at the Baihui (GV 20) and Dazhui (GV 14) acupoints in RSDStreated mice, the hippocampal tissue was subjected to genome-wide gene expression profiling using NGS analysis. Gene expression in the mouse hippocampus after EA treatment under RSDS is shown in the heat map representation (Fig. 6A). Gene expression with $p$-value $\leq 0.05$, and $\geq$ twofold changes were considered significantly differentially expressed. Induced gene sets are presented in red, and repressed gene sets are presented in green
(Fig. 6A). A volcano plot of gene expression with $p<0.05$ (fold change in relative expression as determined by $\log 2$, with upregulated genes are shown in red spots and downregulated genes in blue spots) in the control group versus the RSDS mice (Fig. 6B), and RSDS mice versus RSDS mice treated with EA stimulation (Fig. 6C). Analysis of the differentially expressed genes (DEGs) regulated by RSDS pointed out Ggcx, Gm10222, Rnf181 and Lipocalin 2 (Lcn2) were significantly upregulated and three gene (F5, Rps27, and Srsf1) were downregulated, compared with control group (Additional file 4: Fig. S4A). However, there were five genes (Gm11637, Usp39, $R b s 6 \mathrm{kb2}$, Tmem 72 , and F5) were significantly upregulated, and eighteen genes were downregulated by EA treatment compared with the RSDS group (Additional file 4: Fig. S4B). Remarkably, among the eighteen genes only two genes (Gm10222 and Lcn2) were significantly upregulated by RSDS, but downregulated by EA treatment (Additional file 4: Fig. S4A and B). Particularly, protein-interaction network analysis, as analyzed by the size and depth of each node which is positively correlated to the degree of protein connection to others, identified the $L c n 2$ as the major relevant signaling molecule (Additional file 5: Fig. S5). We focused on one inducible molecule: lipocalin 2 , which secreted by reactive astrocytes was upregulated in the hippocampus following psychological stress [41]. We also found the distribution of $L c n 2$ transcripts using a volcano plot in which $L c n 2$ was upregulated in the RSDS group compared with the control group (Fig. 6B). In contrast, expression of the Lcn2 gene was reduced following EA treatment in the RSDS group (Fig. 6C). As presented in Additional file 6: Fig. S6, the top five pathways enriched from KEGG database were found the subset genes S100A8 (calgranulin A or migration inhibitory factor-related protein 8; MRP8) and S100A9 (calgranulin B, or MRP-14) of Lcn 2 and which relevant pathway: interleukin (IL)-17 signaling pathway (Additional file 6: Fig. S6). Interestingly, modulating transcriptome including S100a 8 and S100a9 genes have been observed that associated with behavioral response to chronic mild stress stimulus [42]. In addition, the hippocampal transcriptome of mice subjected 


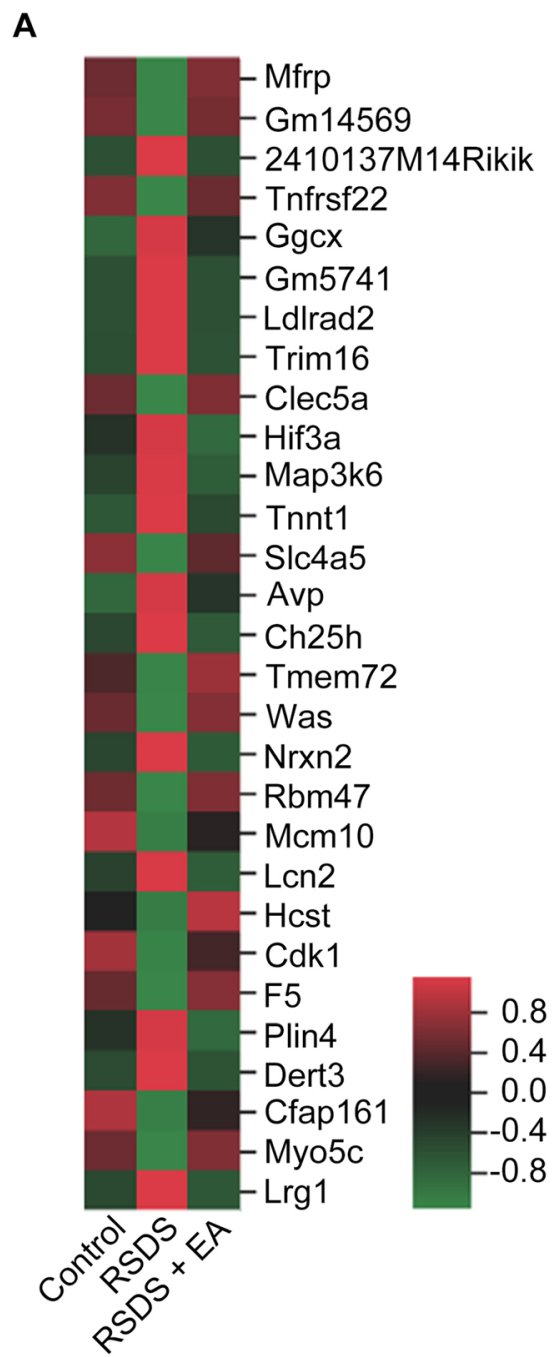

D

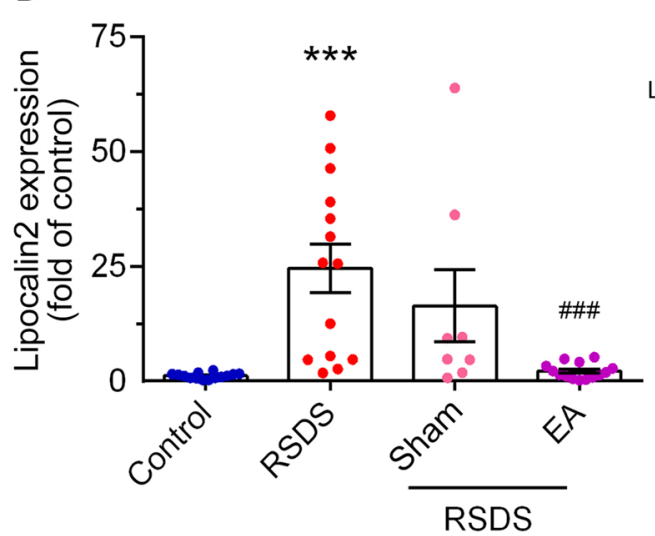

B

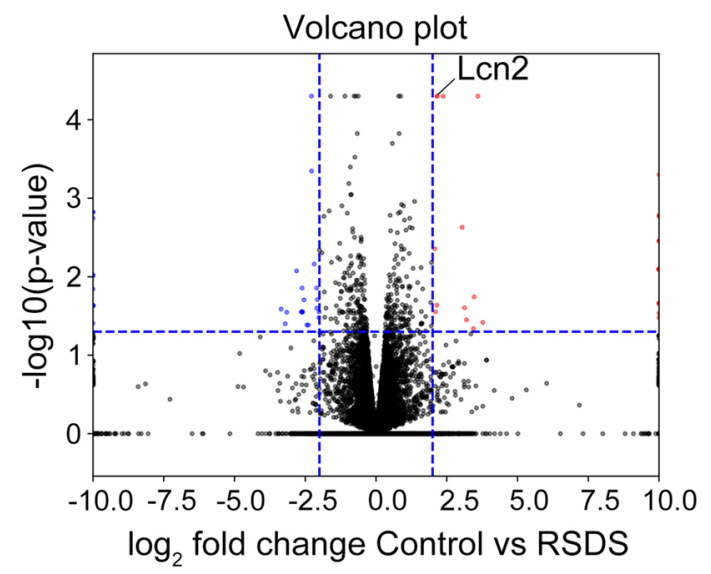

C

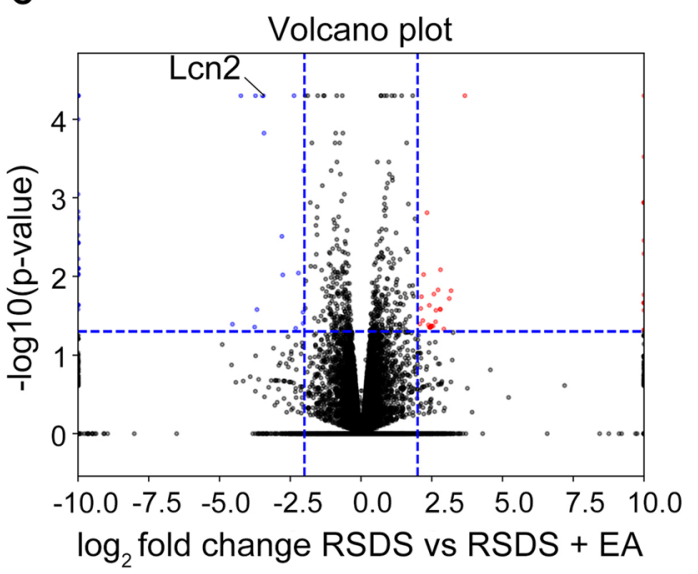

- Down-regulated genes - Up-regulated genes

E

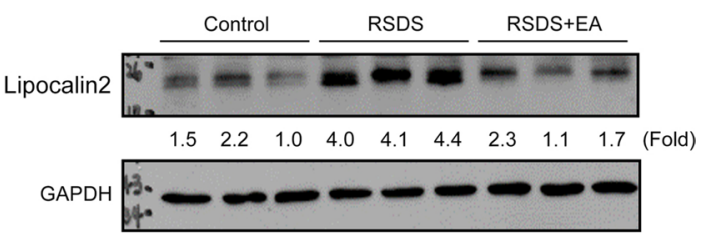

Fig. 6 (See legend on previous page.) 
(See figure on next page.)

Fig. 7 EA stimulation at the Baihui (GV 20) and Dazhui (GV 14) acupoints down-regulates the expression of lipocalin 2 in reactive astrocytes in RSDS-treated mice. Double-labeling immunofluorescence of lipocalin 2 (green) and GFAP (red) in the control (a-e), RSDS (f-j) and RSDS-treated mice with EA stimulation ( $\mathbf{k}-\mathbf{o}$ ) in the ventral hippocampus of the brain sections. DAPI staining (blue) was used to label the nuclei. The merged yellow images indicate co-localization of lipocalin 2 and GFAP (white arrowheads). ( 3 animals in each group) All images were taken at a primary magnification of $200 \times$, and the high magnification (1000x) of $\mathbf{d}, \mathbf{i}$ and $\mathbf{n}$ are shown in $\mathbf{e}, \mathbf{j}$, and $\mathbf{o}$, respectively. EA: electroacupuncture, RSDS: repeated social defeat stress, GFAP: glial fibrillary acidic protein, LCN2: lipocalin 2

to chronic social stress also pointed out that S100a8 and S100a9 genes affected inflammation were upregulated in the hippocampus [43]. Importantly, the immuneresponse genes of Lcn2, S100a8, and S100a9 have been found that markedly increased in the patients' hippocampus with Alzheimer's disease [44]. Recently, progressive multiple sclerosis model caused depression- and anxietylike behaviors, which has been found simultaneously upregulation of IL-17 and GFAP in the hippocampus but not prefrontal cortex [45]. The recent study also reported that cumulative mild stress promoted depression-like behaviors in young adult mice and specifically upregulated IL-17 expression in the hippocampus [46]. We then further verified whether lipocalin 2 expression in RSDS was reduced by EA stimulation. Our results showed that RSDS-induced the upregulation of lipocalin 2 mRNA (Fig. 6D; RSDS vs. control, $p<0.001$; one-way ANOVA) and protein expression (Fig. 6E) in the hippocampus of RSDS mice compared to those in the control group. Conversely, the enhancement of lipocalin 2 was dramatically reduced in the EA group compared to that in the RSDS mice (Fig. 6D; RSDS + EA vs. RSDS, $p<0.001$; one-way ANOVA). In addition, lipocalin 2 levels in the hippocampus were not significantly different between the RSDS and sham treatment groups (Fig. 6D, RSDS + sham vs. RSDS, NS; one-way ANOVA).

Next, we assessed the distribution of lipocalin 2 expression by double labeling for immunofluorescence, counterstained with DAPI (Fig. 7a, f, k), and analyzed by confocal microscopy. There was a low expression of GFAP (Fig. 7b) and lipocalin 2 (Fig. 7c-e) staining in the ventral hippocampus of the mice in the control group. Importantly, both the increase in GFAP (Fig. $7 \mathrm{~g}$ ) and lipocalin 2 (Fig. 7h and i) staining were observed in the RSDS group. Moreover, the increase lipocalin 2 was colocalized in GFAP-positive astrocytes (approximately 3.6-fold) in the ventral hippocampus in the RSDS group (Fig. $7 \mathrm{i}$ and $\mathrm{j}$ ). In contrast, EA stimulation decreased GFAP (Fig. 7l) and lipocalin 2 (Fig. $7 \mathrm{~m}$ ) staining in the ventral hippocampus. Importantly, the co-localization of lipocalin 2 and GFAP was also reduced to approximately $19.7 \%$ by EA stimulation (Fig. $7 \mathrm{n}$ and o). Collectively, our results suggest that lipocalin 2 may be a key regulatory factor for EA treatment in RSDS mice.

\section{Overexpression of lipocalin 2 protein in the brain causes social avoidance and anxiety-like behaviors}

To determine whether increasing lipocalin 2 in the brain associates RSDS-induced negative behaviors, C57/BL6 mice received an i.c.v. injection of mouse recombinant lipocalin 2 protein on a stereotaxic apparatus (Fig. 8A, left panel), and behavioral tasks were measured after 6 days. The right panel of Fig. 8A shows the representative heat maps of the social interaction analysis following RSDS. In the social interaction test, the time spent in the social interaction zone (Fig. 8B) and the social interaction ratio (Fig. 8D) were significantly reduced in lipocalin 2-injected mice compared to vehicle-injected mice. Conversely, the lipocalin 2 injected mice had a tendency to increase the time spent in the corner (Fig. 8E). In addition, there was no significant difference in the time spent in the social interaction zone (Fig. 8C) or corners (Fig. 8F), with the target mice being absent. The velocity traveled by both the lipocalin 2 - and vehicle-injected mice was also not significantly different (Fig. 8G). Figure 9A shows the representative heat maps of the light compartment in the L/D box task. The lipocalin 2-injected mice had a low frequency when traveling in the light compartment, compared with the vehicleinjected mice (Fig. 9B; lipocalin 2 vs. vehicle, $p<0.05$; Student's t-test). Surprisingly, lipocalin 2-injected mice had a longer latency to the first entry into the light compartment (Fig. 9C; lipocalin 2 vs. vehicle, $p<0.05$; Student's t-test). Moreover, the cumulative time spent in the light compartment was decreased in the lipocalin 2-injected mice compared to the vehicle group (Fig. 9D; lipocalin 2 vs. vehicle, $p<0.05$; Student's t-test). In addition, the velocities traveled by these two groups were not significantly different (Fig. 9E). We also performed the EPM task to further determine cognitive impairment in lipocalin 2 injected mice. As shown in Fig. 10A, lipocalin 2-treated mice spent less time in the open arms than the vehicle group (lipocalin 2 vs. vehicle, $p<0.01$; Student's t-test). In parallel, the lipocalin 2-injected mice spent more time in the closed arms than the vehicle-injected mice (Fig. 10B, lipocalin 2 vs. vehicle, $p<0.01$; Student's $\mathrm{t}$-test). In addition, there were no significant differences observed in the time spent in the center (Fig. 10C) and the velocity traveled (Fig. 10D) in the EPM apparatus in both groups. Collectively, our results suggest that 


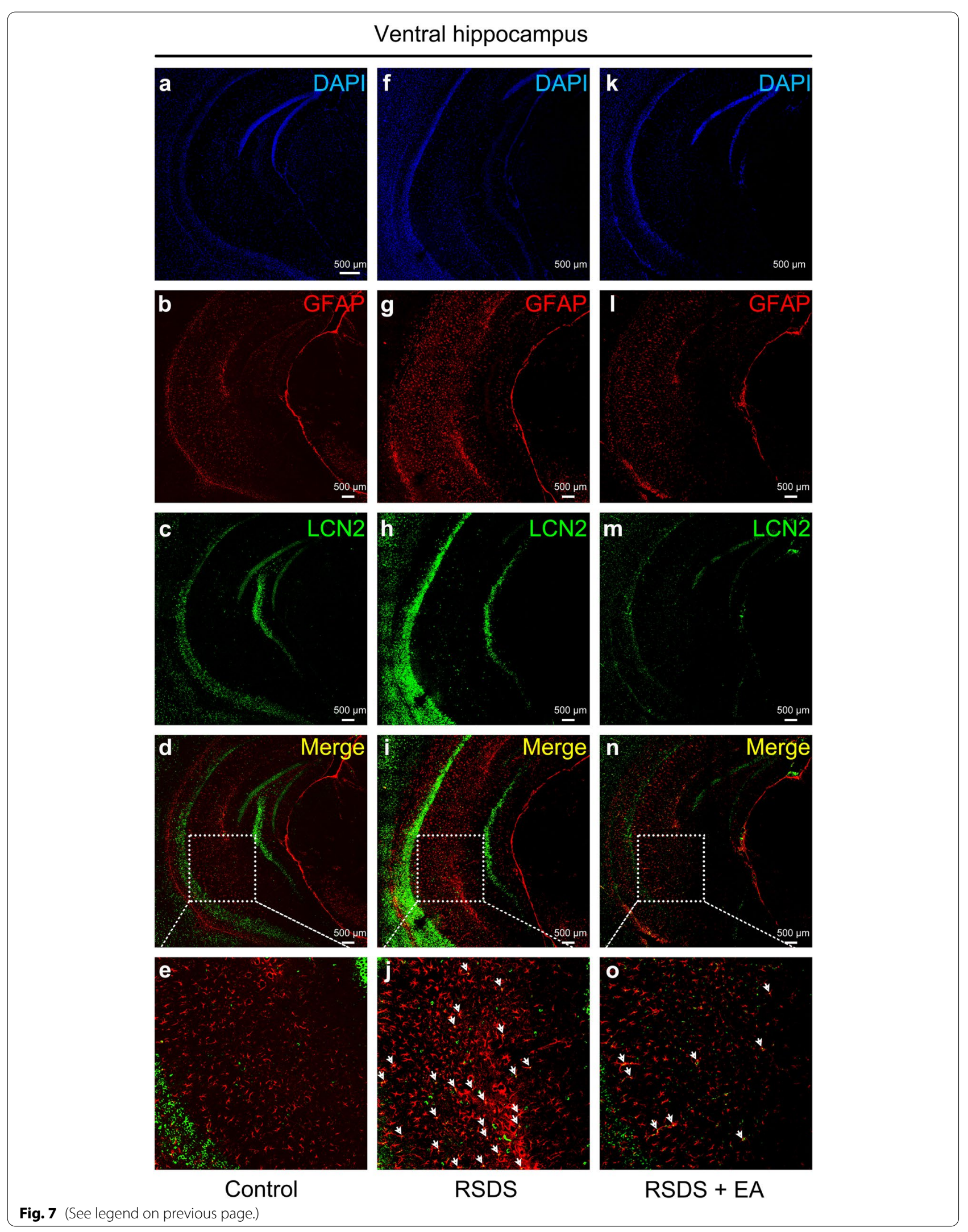


(See figure on next page.)

Fig. 8 Overexpression of lipocalin 2 in the brain induces social avoidance. Single i.c.v. injection of recombinant lipocalin 2 protein $(7 \mu \mathrm{L}$ and $1 \mu \mathrm{g} /$ $\mathrm{mL}$ ) or vehicle in the brain of the mice and social interaction behavioral tests were performed after 6 days. A i.c.v. injection position in lateral ventricles (left panel), and the representative heat maps were shown in right panel. The time spent in the interaction zone while the target was present (B) or absent (C). D The social interaction ratio (the time spent in the interaction zone while the target was absent / the time spent in the interaction zone while the target was present). The time spent in corners while the interaction zone with (E) or without (F) the target mice. G The velocity traveled in the target apparatus. Quantitative data are presented as the mean \pm SEM ( $n=5$ each group). The Student's t-test was used to examine the significance of the mean. ${ }^{*} p<0.05$ vs. the vehicle or control groups. i.c.v.: intracerebroventricular, LCN2: lipocalin 2, NS: not significant, SEM: standard error of mean

lipocalin 2 overexpression in the brain may provoke cognitive impairment, including social avoidance and anxiety-like behaviors, similar to RSDS-treated mice.

\section{Overexpression of lipocalin 2 protein in the brain alters astrocyte activation and stress-related gene expression} We further assessed whether increasing lipocalin 2 in brain alters the activation of astrocytes and stress-related genes. Astrocytes in the lipocalin 2 group showed morphological characteristics of activation in the dorsal (Fig. 11A) or ventral (Fig. 11B) hippocampus after the mice received i.c.v. injection of mouse recombinant lipocalin 2 protein. The quantitative results showed that the GFAP immunoreactivity was significantly increased in both the dorsal (Fig. 11C, lipocalin 2 vs. vehicle, $p<0.01$; Student's t-test) and ventral (Fig. 11D, lipocalin 2 vs. vehicle, $p<0.05$; Student's t-test) hippocampal CA3 region in the lipocalin 2 -injected mice as compared to the vehicle group. Furthermore, we examined whether increasing lipocalin 2 alters stress-related gene expression in the hippocampus. As shown in Fig. 12, the expression of MR (lipocalin 2 vs. vehicle, $p<0.01$; Student's t-test) or GR (lipocalin 2 vs. vehicle, $p<0.0001$; Student's t-test) was significantly lower in the lipocalin 2 group than in the vehicle group. On the other hand, the expression of MAO-A (lipocalin 2 vs. vehicle, $p<0.01$; Student's t-test) or MAO-B (lipocalin 2 vs. vehicle, $p<0.05$; Student's t-test) was dramatically increased in the lipocalin 2 group compared to the vehicle group. Collectively, these results indicate that increasing lipocalin 2 in the brain promotes astrocyte activation and alters stress-related gene expression in the hippocampus, which may cause behavioral impairment.

\section{Discussion}

Acupoint specificity means acupuncture at acupoints induces a more efficient therapeutic response than nonacupoint. Particularly, there are three properties of special structures beneath acupoints have been supported including muscle-spindle-rich area, cutaneous-receptor rich area, and tendon-organ-rich area [47]. The proportional locations of the acupoints in mouse in this study were determined following the anatomical description in the WHO guidelines for human acupoints [48, 49]. There are only few studies have reported the effects of EA treatment in an animal model of sociability. Laser acupuncture at Shenmen (HT7) in a postnatal autistic mouse model improved autistic-like behaviors, including learning, memory, and social behavior [50]. Moreover, stimulation at the catgut acupoint in a social isolation mouse model is effective in reversing depression-like behavior [51]. EA treatment at the Zusanli (ST36) acupoint has also been reported to reverse the memory impairments of isolated mice [52], and to improve the social behaviors in the low socially interacting rats [53]. EA has previously reported to improve PTSD-like behaviors in rats by enhancing hippocampal endocannabinoid signaling [54]. Recently, several preclinical evidence indicated that Baihui (GV20) acupoint is most frequently used in PTSD [27]. In Chinese medicine theory, these acupoints are on the "Du meridian", involves direct communication with the brain. Moreover, the Dazhui (GV14) and Baihui (GV20) commonly used acupoints in traditional Chinese medicine to treat neurological disorders. The stimulation of EA at the Dazhui (GV14) and Baihui (GV20) acupoints exerts neuroprotective effects in cerebral ischemia-reperfusion injury in rats $[55,56]$. In a rodent model, EA treatment at the Dazhui (GV14) and Baihui (GV20) acupoints also exerts neuroprotective effects in cerebral ischemia-reperfusion injuries $[55,56]$. In the clinic, acupuncture treatment at the Dazhui (GV14) and Baihui (GV20) acupoints effectively improved the symptoms of infantile primary and Jacksonian epilepsy $[57,58]$. In the present study, EA treatment at the Dazhui (GV14) and Baihui (GV20) acupoints reduced RSDS-induced anxiety-like behaviors and social avoidance, which possess potent therapeutic effects in mood disorders. Importantly, our results also support previous studies that stimulation of bilateral Tianzong (SI11) acupoints could be sham acupoints in an acupuncture study.

Lipocalin 2 (also known as neutrophil gelatinase-associated lipocalin [NGAL]) in the CNS is predominantly secreted by astrocytes under inflammatory conditions and promotes morphological transformation and migration in astrocytes [59]. Moreover, lipocalin 2 has been reported to be released by injured neurons as a 

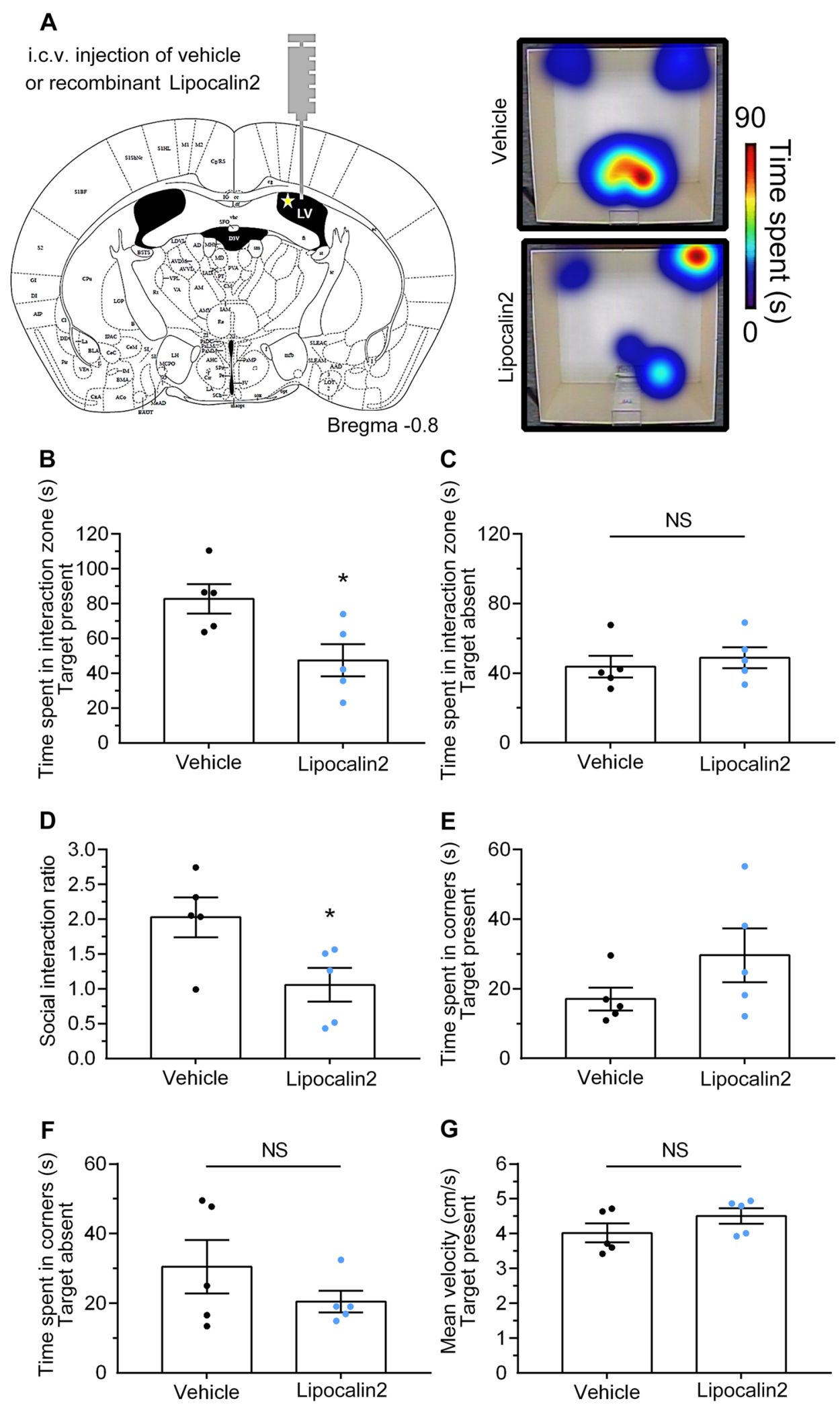

Fig. 8 (See legend on previous page.) 
A

Light zone

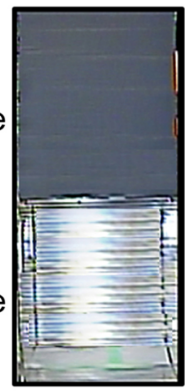

Apparatus

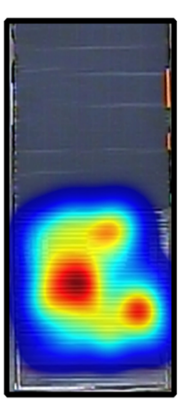

Vehicle

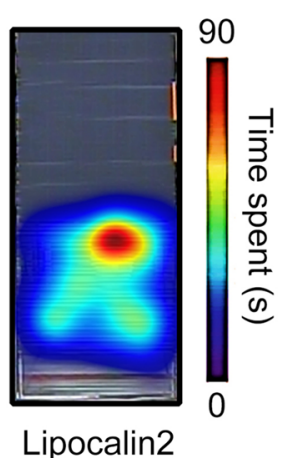

Lipocalin2

90

군
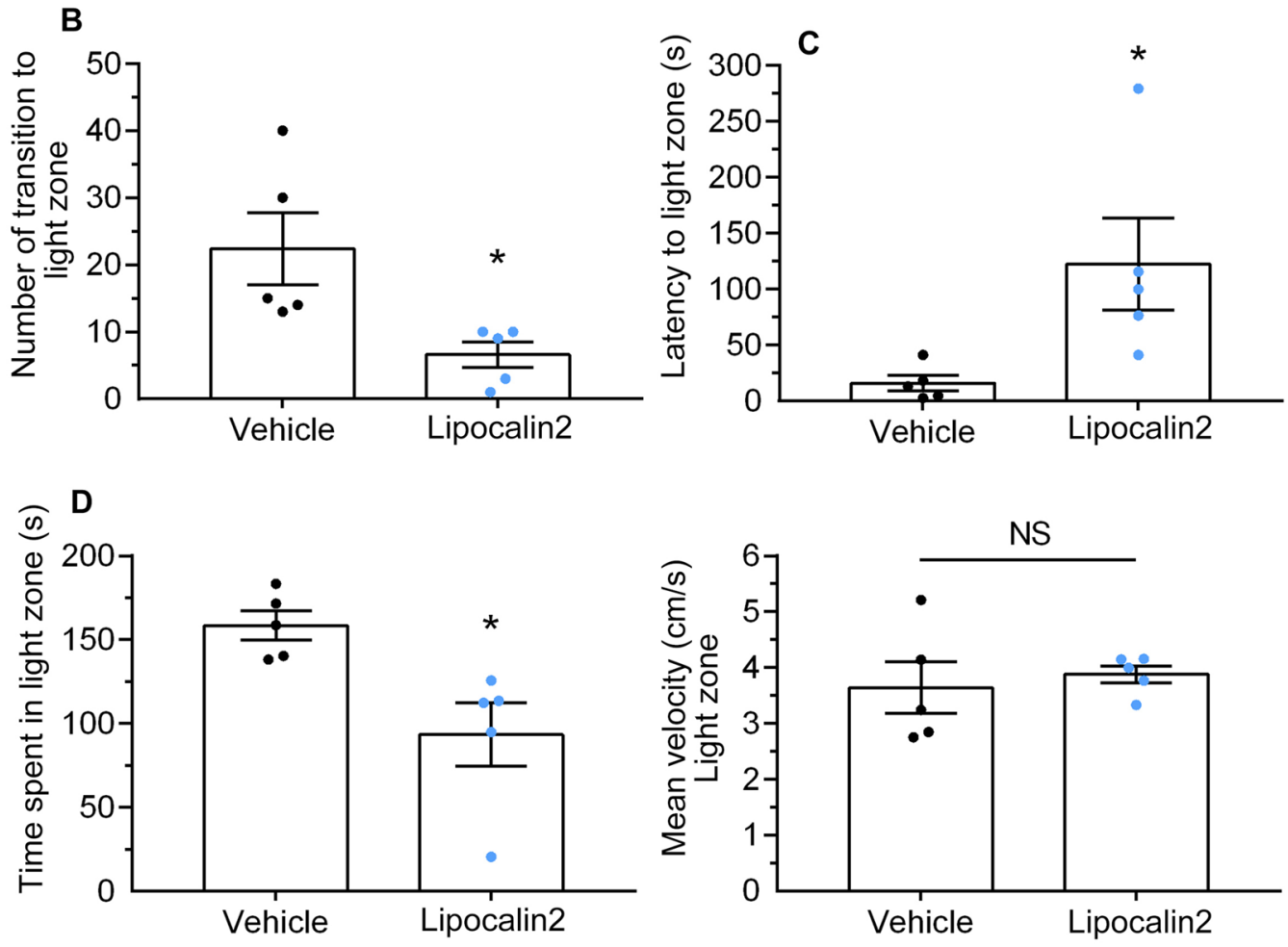

Fig. 9 Overexpression of lipocalin 2 in the brain induces anxiety-like behaviors in the light and dark task. Single intracerebroventricular injection of recombinant lipocalin 2 protein $(7 \mu \mathrm{L}, 1 \mu \mathrm{g} / \mathrm{mL})$ or vehicle in the brain of the mouse, and light and dark tasks were assessed after 6 days. A Representative heat maps for the activities of each group during the light-dark test. The frequency during the time spent in the light compartment (B), the latency to the first entry into the light compartment (C), or the cumulative time in the light compartment (D) were determined. The velocity traveled in the light compartment is measured in (E). Quantitative data are presented as mean \pm SEM ( $n=5$ per group). The Student's t-test was used to examine the significance of the mean. ${ }^{*} p<0.01$, vs. the vehicle or control group. LCN2: lipocalin 2, NS: not significant, SEM: standard error of mean

"help-me" distress signal that activates the astrocytes into potentially pro-recovery phenotypes [60]. Recently, lipocalin 2 has been shown to play an important role in pathological conditions, specifically its association with cognition during the progression of pathology [61]. Importantly, lipocalin 2 has previously been found to be upregulated in the hippocampus following psychological stress and regulates stress-induced neuronal excitability and anxious behavior [62], which has been considered as a potential biomarker in agingrelated cognitive decline [63]. Lipocalin 2-null mice are reported to display anxious and depressive-like behaviors, as well as cognitive and memory impairment [64]. A recent report also suggests that lipocalin 2 secreted from reactive astrocytes in the cerebrospinal fluid is a promising biochemical marker candidate for the differential diagnosis of vascular dementia and neurodegenerative dementias [65]. A recent study also reported 

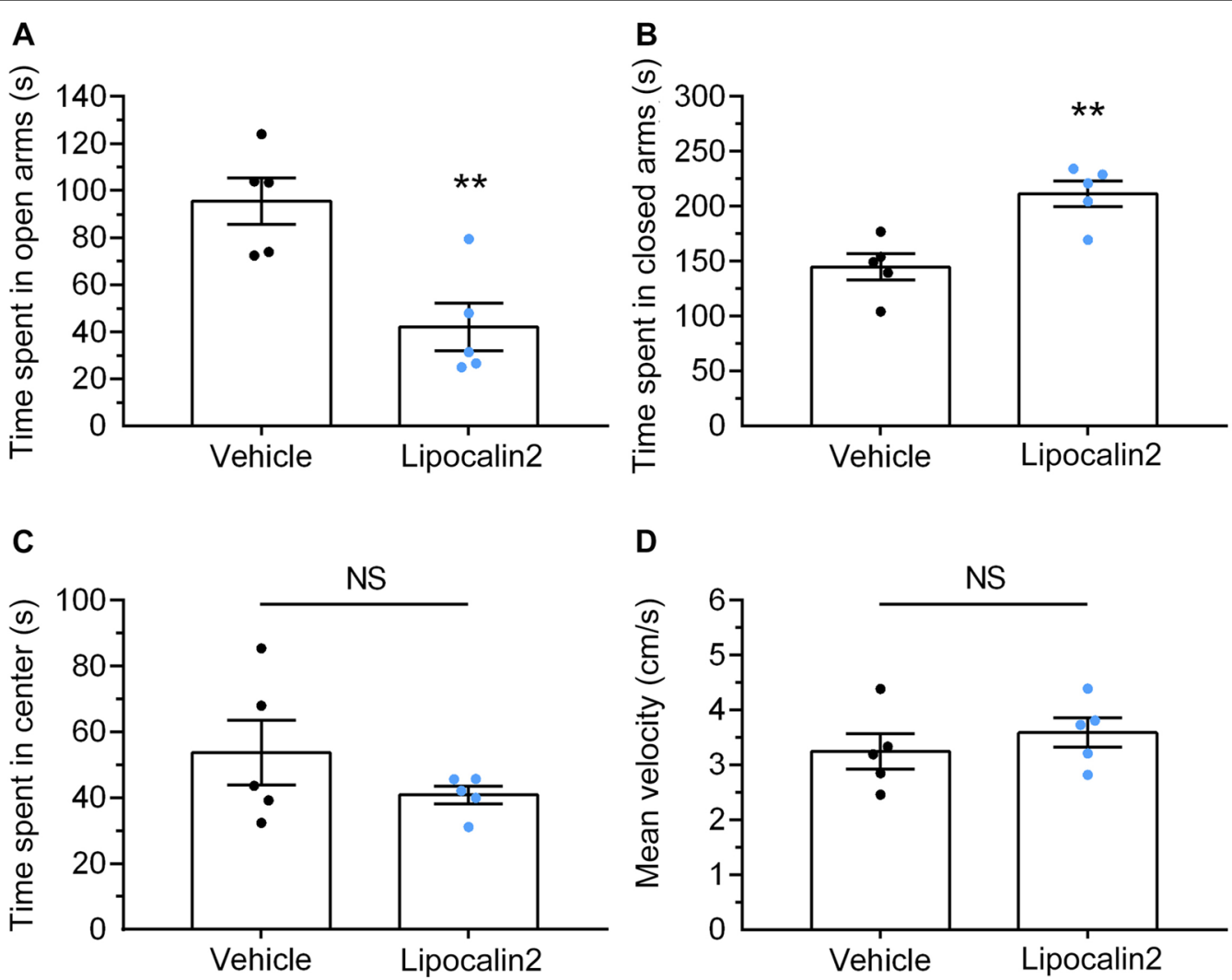

Fig. 10 Overexpression of lipocalin 2 in the brain induces anxiety-like behaviors in the EPM task. Single intracerebroventricular injection of recombinant lipocalin 2 protein $(7 \mu \mathrm{L}, 1 \mu \mathrm{g} / \mathrm{mL})$ or vehicle in the brain of the mice and the EPM task were performed after 6 days. Time spent in the open arms (A), closed arms (B), or center (C) of the EPM apparatus. D Velocity traveled in the EPM apparatus. Quantitative data are presented as mean \pm SEM ( $n=5$ per group). The Student's t-test was used to examine the significance of the mean. ${ }^{* *} p<0.01$ vs. the vehicle group. EPM: elevated plus maze, LCN2: lipocalin 2, NS: not significant, SEM: standard error of mean

that astrocytes from patients with Parkinson's disease are more responsive to lipocalin 2 stimulation for reactive astrocytosis [66]. Higher lipocalin 2 expression in patients brain has been found that significantly associated with depression scores [67]. Moreover, elevated plasma lipocalin 2 levels is also been reported that associated in depressed older patients with a pathological waist circumference [68]. Interestingly, lipocalin 2 upregulation has also been reported that associated with sex-specific cognitive functioning in late-life depression [69]. Our results revealed that astrocyte activation and lipocalin 2 expression induced by RSDS were effectively reduced by EA treatment at the Baihui (GV 20) and Dazhui (GV 14) acupoints. Importantly, overexpression of lipocalin 2 in the brain also elicited PTSD-like behaviors, such as social avoidance and anxiety-like behaviors. Our findings support the previous reports that the expression of lipocalin 2 in the brain may be an important regulator of cognitive symptoms, and increased lipocalin 2 levels is associated with depression or PTSD.
The hippocampus is particularly vulnerable to chronic stress, if impaired, leading to mood disorders such as anxiety-like and depressive-like behaviors [70]. There are at least two distinct functional regions of the hippocampus, namely the ventral and dorsal hippocampi. The dorsal region serves for memory and learning of conceptual information, but the ventral hippocampus primarily modulates emotional processes, such as fear and anxiety [71]. Particularly, ventral hippocampus has also been reported which is a specific region regulates susceptibility to chronic social defeat stress [72], and may act as a potential key mediator of stress-induced anxiety-like behavior [73]. Surprisingly, the lipocalin 2 null mice have been found that the ventral hippocampus were hypertrophic and display anxious and depressive-like behaviors [64]. The dorsal hippocampal CA1 neural systems have also been reported to modulate anxiety behaviors [74]. Remarkably, the hippocampal CA3 region is a crucial role in the stress response that exerts a negative feedback in the regulation of the HPA axis [75]. In addition, 
A
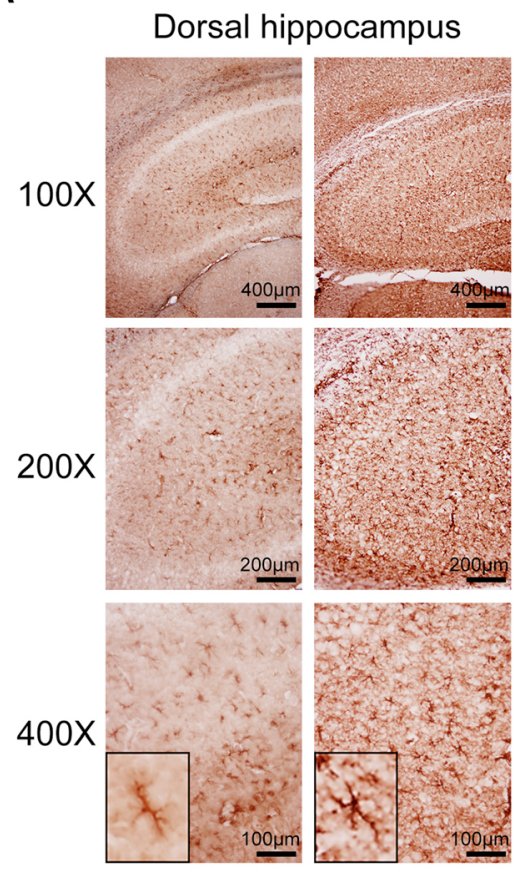

Vehicle

C

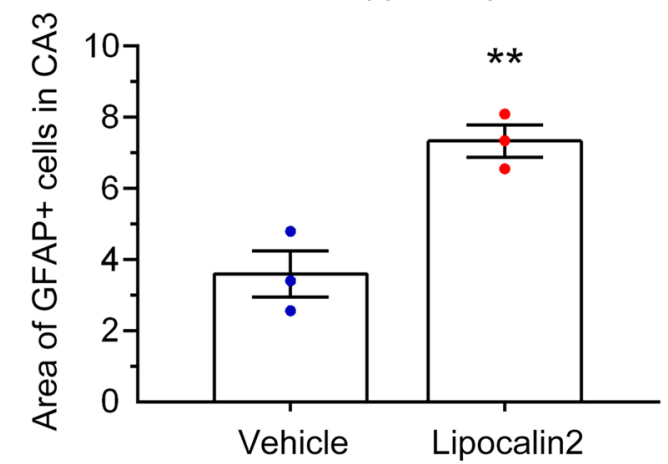

B

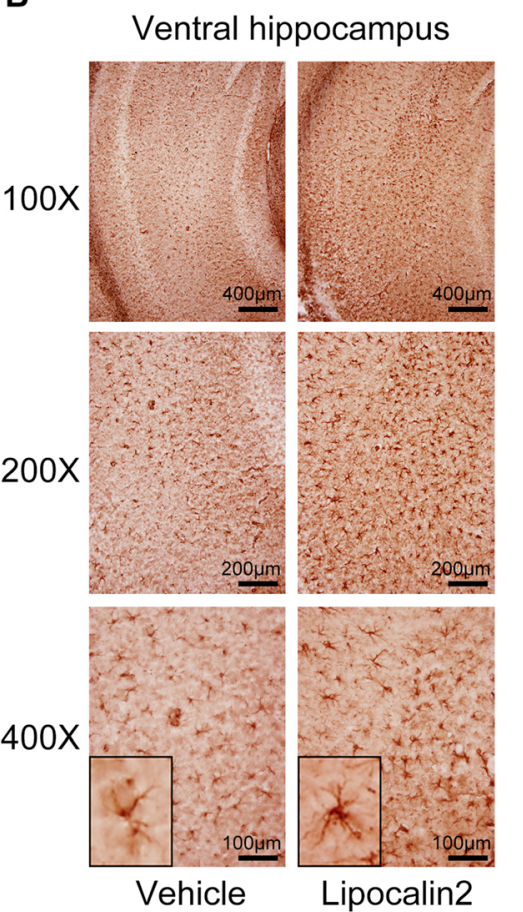

D

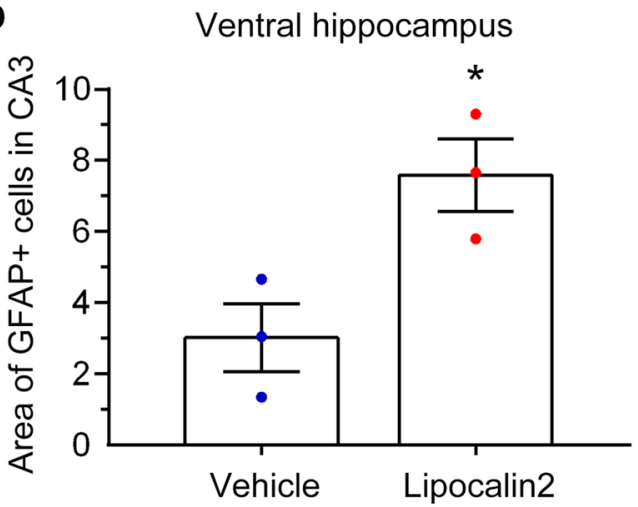

Fig. 11 Overexpression of lipocalin 2 in the brain induces astrocyte activation in the hippocampus. After a single intracerebroventricular injection of recombinant lipocalin 2 protein $(7 \mu \mathrm{L}, 1 \mu \mathrm{g} / \mathrm{mL})$ or vehicle in the brain of the mice for 7 days, the brain coronal slices were fixed for immunohistochemistry with anti-GFAP antibody. Representative images were taken from the dorsal (A) and ventral (B) hippocampal CA3 areas, and the GFAP immunoreactivity was quantified as the percentage of GFAP-positive cells in the dorsal (C) and ventral (D) hippocampi of the vehicle and lipocalin 2 groups. Quantitative results are presented as mean \pm SEM ( $n=3$ per group). The Student's t-test was used to examine the significance of the mean. ${ }^{*} p<0.05$ vs. the vehicle group. ${ }^{* *} p<0.01$ vs. the vehicle group. GFAP: glial fibrillary acidic protein, LCN2: lipocalin 2, SEM: standard error of mean

the hippocampal CA3 is also a region of dynamic stressinduced structural plasticity [76]. Chronic stress has been proposed to induce hippocampal CA3 neuronal retraction, which then impairs activity of the HPA axis, leading to disturbed glucocorticoid release [77]. Particularly, chronic glucocorticoid treated animals have also been observed that remodeling the CA3 pyramidal neurons but not the CA1 pyramidal cells or the granule cells of the dentate gyrus [78]. Moreover, CA3 region has been observed that is more vulnerable and experts earlier response of adhesion properties that cerebral cortex and CA1 region under ischemia challenge [79]. It has also been reported that stress-induced dendritic retraction of CA3 pyramidal neurons has been implicated as mechanisms contributing to hippocampal shrinkage [80]. 


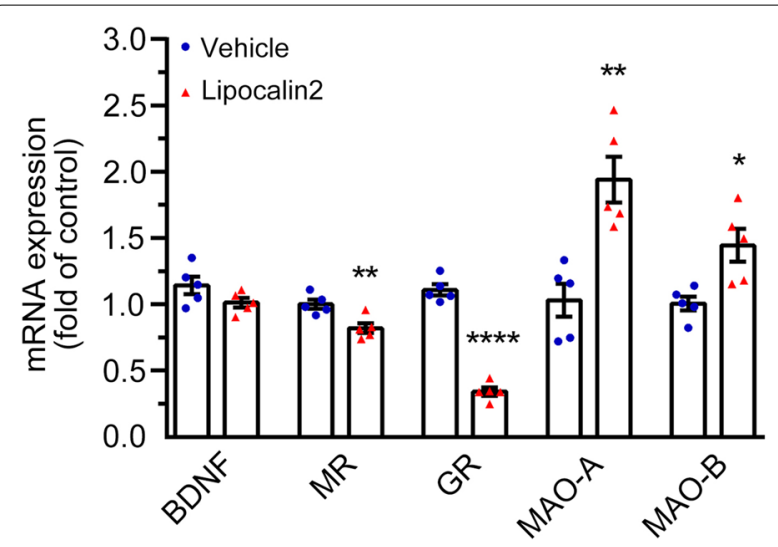

Fig. 12 Overexpression of lipocalin 2 in the brain alters stress-related gene expression in the hippocampus. After a single intracerebroventricular injection of recombinant lipocalin 2 protein $(7 \mu \mathrm{L}, 1 \mu \mathrm{g} / \mathrm{mL})$ or vehicle in the brain of the mice for 7 days, the hippocampal tissues were collected for mRNA assay. The expression of $M R$, GR, MAO-A, or MAO-B was determined by real-time PCR. Quantitative data are presented as mean $\pm \operatorname{SEM}(n=5$ per group). The Student's t-test was used to examine the significance of the mean. ${ }^{*} p<0.05$ vs. the vehicle group. ${ }^{* *} p<0.01$ vs. the vehicle group. ${ }^{* * * *} p<0.001$ vs. the vehicle group. GR: glucocorticoid receptor, MAO-A: monoamine oxidase A, MAO-B: monoamine oxidase B, MR: mineralocorticoid receptor, $\mathrm{PCR}$ : polymerase chain reaction, SEM: standard error of mean

Decreased levels of endogenous anti-inflammatory proteins Nrf2 and Keap1 in the CA3 hippocampus of mice have been implicated in the pathophysiology of depression after social defeat stress [81]. Moreover, infusion of BDNF recombinant protein or a TrkB agonist into the CA3 hippocampus promoted long-lasting antidepressant effects in mice models of depression [82]. Otherwise, Nrf2 knockout mice have been found that reveal lower BDNF and its receptor TrkB signaling and higher inflammation in the CA3, which also show depression-like phenotypes than those of WT mice [83]. Single prolonged stress-enhanced astrocyte activation in the rat brain could last a few days, which increasing the vulnerability of abnormal fear and learning [84]. GFAP expression in brain may suggest a response of exposure to stress [85]. Remarkably, GFAP has been reported that plays a crucial role in CA3 neuronal survival after injury [86]. Importantly, the populations of astrocytes have been observed that increased in the CA3 hippocampus after spatial learning in rats [87]. Furthermore, the neonatal mice subjected to maternal separation presented an increased activation of astrocytes in the CA3 area [88]. The activity-stress rats have been observed increased significantly GFAP-immunoreactive cells in the hippocampal CA3 region, which is similar to the response found in ischemia [89]. Therefore, this study has focused on a specific marker expression in the vulnerable brain region responds to social-defeat stress challenge to access whether EA treatment improves RSDS-responsive expression of GFAP and astrocyte-associated lipocalin 2 in the hippocampal CA3 region.

As a general rule, a malfunction of the HPA axis has been strongly implicated in the development of mood disorders under stress [90]. There are two types of adrenal steroid receptors in the hippocampus: MR and GR. MR plays an important role in negative feedback and affects hippocampal function in patients with PTSD [91]. Furthermore, downregulation or disruption of GR expression in the hippocampus has been found to enhance depression and anxiety behaviors [92]. Our previous results showed that the injection of a GR antagonist impaired social behavior [11]. On the other hand, treatment options for depressive and anxious patients are based on the monoamine hypothesis and aim to increase the availability of monoamines [93]. Both MAO-A and MAO-B modulate neurobiochemistry by degrading monoamine neurotransmitters, including serotonin, dopamine, and norepinephrine [94]. A significant increase in the monoamine oxidase MAO-A is found in association with the pathogenesis of major depressive disorders [95], and selective serotonin reuptake inhibitor treatment elevated MAO-A binding in the brain regions of patients with depression, which contributes to recurrence [96]. In previous studies, C57BL/6 mice were subjected to the RSDS model to induce social avoidance and chronic anxiety-like behaviors, and alter the expression of stress-related genes in the brain [97]. Our previous study also reported that chronic social defeat stress induces social avoidance behavior and increases MAO-A and MAO-B expression in mice [11]. The present study found that overexpression of lipocalin 2 in the brain evoked PTSD-like behaviors such as social avoidance and anxiety-like behaviors. These results also found that overexpression of lipocalin 2 in the brain induced astrocyte activation, decreased $M R$ and GR, and increased the expression of MAO-A and MAO-B.

\section{Conclusions}

We established a preclinical repeated social defeat mouse model, which develops symptoms of PTSD similar to humans, such as social avoidance, despair, and anxiety. The present study suggests that the stimulation of EA, but not needle insertion at the Baihui (GV 20) and Dazhui (GV 14) acupoints effectively improves RSDS-elicited social avoidance and anxiety-like behaviors. Moreover, astrocyte activation and lipocalin 2 expression induced by RSDS were effectively reduced by EA treatment at the Baihui (GV 20) and Dazhui (GV 14) acupoints. Furthermore, overexpression of lipocalin 2 in the brain also elicited PTSD-like behaviors, such as social avoidance 
and anxiety-like behaviors. Our findings suggest that EA stimulation at the Baihui (GV 20) and Dazhui (GV 14) acupoints effectively improves RSDS-elicited social avoidance and anxiety-like behaviors, and upregulation of lipocalin 2 in the brain may be an important biomarker for the development of PTSD-related symptoms.

\section{Abbreviations}

PTSD: Post-traumatic stress disorder; EA: Electroacupuncture; CNS: Central nervous system; RSDS: Repeated social defeat stress; IL: Interleukin; TNF: Tumor necrosis factor; GFP: Green fluorescent protein; EPM: Elevated plus maze; L/D: Light and dark; GFAP: Glial fibrillary acidic protein; PCR: Polymerase chain reaction; MR: Mineralocorticoid receptor; GR: Glucocorticoid receptor; Ct: Cycle threshold; ANOVA: Analysis of variance; HPA: Hypothalamus-pituitary-adrenal.

\section{Supplementary Information}

The online version contains supplementary material available at https://doi. org/10.1186/s13041-021-00860-0.

Additional file 1: Fig. S1. Effects of electroacupuncture stimulation at the bilateral Tianzong (SI1 1) acupoints in the social interaction task. After RSDS, the experimental mice were introduced into the social interaction test apparatus. The time spent in the interaction zone was determined while the target was present (A) or absent (B). (C) The social interaction ratio was calculated by dividing the time spent in the interaction zone with the target present by the time spent in the interaction zone with the target absent. The time spent in the corners was determined by the interaction zone with the target (D) or without the target (E). Quantitative data are presented as the mean \pm SEM ( $n=5$ each group). One-way ANOVA with a post-hoc Tukey's test was used to examine the significance of the mean. ${ }^{* *} p<0.01$ vs. the control group. ${ }^{* * *} p<0.001$ vs. the control group. $\# \# p<0.01$ vs. the RSDS group. ANOVA: analysis of variance, EA: electroacupuncture, NS: not significant, RSDS, repeated social defeat stress, SEM, standard error of mean.

Additional file 2: Fig. S2. Effects of EA stimulation at the bilateral Tianzong (SI11) acupoints in the light-dark task. After RSDS, the experimental mice were placed in a light-dark task apparatus. The frequency spent in the light compartment (A), the latency to the first light compartment (B), or the cumulative time in the light compartment (C) were determined. Quantitative data are presented as the mean \pm SEM ( $n=5$ each group). One-way ANOVA with a post-hoc Tukey's test was used to examine the significance of the mean. ${ }^{* *} p<0.01$ vs. the control group. ${ }^{* *} p<0.001$ vs. the control group. $\# p<0.05$ vs. the RSDS group. ANOVA: analysis of variance, EA: electroacupuncture, RSDS, repeated social defeat stress, SEM, standard error of mean

Additional file 3: Fig. S3. Effects of EA stimulation at the bilateral Tianzong (SI11) acupoints in the EPM task. After RSDS, the experimental mice were introduced into the EPM apparatus. The time spent in the open arms (A) and center (B) of the apparatus were determined. Quantitative data are presented as the mean \pm SEM ( $n=5$ each group). One-way ANOVA with a post-hoc Tukey's test was used to examine the significance of the mean. * $p<0.05$, RSDS group compared to the control group. $\# p<0.05$, EA group compared to the RSDS group. ANOVA: analysis of variance, EPM: elevated plus maze, EA: electroacupuncture, NS: not significant; RSDS, repeated social defeat stress.

Additional file 4: The bar chart of DEG expression. (A) The log2 ratio of DEG expression in RSDS VS Control. (B) The log2 ratio of DEG expression in EA treatment VS RSDS.

Additional file 5: Protein-protein interaction network generated from GeneMANIA analysis. The size of each node presents the degree of connectivity in PPI network, and large node shares more connection to other nodes.

Additional file 6: Top five pathways prediction of DEGs by KEGG.

\section{Acknowledgements}

This work was also partially supported by the Chinese Medicine Research Center of China Medical University, under The Featured Areas Research Center Program within the framework of the Higher Education Sprout Project, the Ministry of Education, Taiwan. The authors thank Ms. Hsin-Yi Chung for the technical support with EA.

\section{Authors' contributions}

YC: investigation, validation, visualization, methodology; SX: formal analysis, investigation, methodology, validation, visualization; CC: methodology, validation; DL: conceptualization, formal analysis, investigation, supervision, validation, visualization, writing original draft \& editing. All authors read and approved the final manuscript.

\section{Funding}

This work was supported in part by grants from the Ministry of Science and Technology, Taiwan (MOST 110-2320-B-039 -028 -MY3, 109-2320-B-039-020 and 108-2320-B-039-015); China Medical University, Taiwan (CMU-S-13, CMU-S-14, and CMU 107-ASIA-16); China Medical University Hosipital, Taiwan (DMR109-65 and DMR109-68).

\section{Availability of data and materials}

The data that support the findings are not publicly available. Data are available from the authors upon reasonable request.

\section{Declarations}

\section{Ethics approval and consent to participate}

All animal experiments were performed in accordance with the Animal Care and Use Guidelines of China Medical University (IACUC Approval No. CMUIACUC-2019-139).

\section{Consent for publication}

Not applicable.

\section{Competing interests}

The authors declare that they have no known competing financial interests or personal relationships that could have appeared to influence the work reported in this paper.

\section{Author details}

${ }^{1}$ Graduate Institute of Acupuncture Science, China Medical University, Taichung, Taiwan. ${ }^{2}$ Department of Pharmacology, School of Medicine, China Medical University, Taichung, Taiwan. ${ }^{3}$ Institute of New Drug Development, China Medical University, Taichung, Taiwan. ${ }^{4}$ Department of Photonics and Communication Engineering, Asia University, Taichung, Taiwan.

Received: 21 June 2021 Accepted: 13 September 2021

Published online: 26 September 2021

\section{References}

1. Guideline Development Panel for the Treatment of Ptsd in Adults APA: summary of the clinical practice guideline for the treatment of posttraumatic stress disorder (PTSD) in adults. Am Psychol. 2019, 74:596-607.

2. Kothgassner OD, Goreis A, Kafka JX, Van Eickels RL, Plener PL, Felnhofer A. Virtual reality exposure therapy for posttraumatic stress disorder (PTSD): a meta-analysis. Eur J Psychotraumatol. 2019;10:1654782.

3. Duman RS, Aghajanian GK. Synaptic dysfunction in depression: potential therapeutic targets. Science. 2012;338:68-72.

4. McEwen BS, Morrison JH. The brain on stress: vulnerability and plasticity of the prefrontal cortex over the life course. Neuron. 2013;79:16-29.

5. Tennant C. Work-related stress and depressive disorders. J Psychosom Res. 2001;51:697-704.

6. Krishnan V, Han MH, Graham DL, Berton O, Renthal W, Russo SJ, Laplant Q Graham A, Lutter M, Lagace DC, et al. Molecular adaptations underlying susceptibility and resistance to social defeat in brain reward regions. Cell. 2007;131:391-404. 
7. Becker C, Zeau B, Rivat C, Blugeot A, Hamon M, Benoliel JJ. Repeated social defeat-induced depression-like behavioral and biological alterations in rats: involvement of cholecystokinin. Mol Psychiatry. 2008;13:1079-92.

8. Golden SA, Covington HE 3rd, Berton O, Russo SJ. A standardized protocol for repeated social defeat stress in mice. Nat Protoc. 2011;6:1183-91.

9. McKim DB, Patterson JM, Wohleb ES, Jarrett BL, Reader BF, Godbout JP, Sheridan JF. Sympathetic release of splenic monocytes promotes recurring anxiety following repeated social defeat. Biol Psychiatry. 2016;79:803-13.

10. Lin HY, Liu YS, Huang CY, Cathomas F, Liu K, Wang J, Cheng HT, Lai SW, Liu YC, Chen CJ, et al. SUMO E3 ligase PIAS1 is a potential biomarker indicating stress susceptibility. Psychoneuroendocrinology. 2020;120:104800

11. Lin HY, Liu YS, Liu YC, Chen CJ, Lu DY. Targeted ubiquitin-proteasomal proteolysis pathway in chronic social defeat stress. J Proteome Res. 2019;18:182-90.

12. Walker AK, Kavelaars A, Heijnen CJ, Dantzer R. Neuroinflammation and comorbidity of pain and depression. Pharmacol Rev. 2014;66:80-101.

13. Setiawan E, Wilson AA, Mizrahi R, Rusjan PM, Miler L, Rajkowska G, Suridjan I, Kennedy JL, Rekkas PV, Houle S, Meyer JH. Role of translocator protein density, a marker of neuroinflammation, in the brain during major depressive episodes. JAMA Psychiat. 2015;72:268-75.

14. Niraula A, Wang Y, Godbout JP, Sheridan JF. Corticosterone production during repeated social defeat causes monocyte mobilization from the bone marrow, glucocorticoid resistance, and neurovascular adhesion molecule expression. J Neurosci. 2018;38:2328-40.

15. Savignac HM, Hyland NP, Dinan TG, Cryan JF. The effects of repeated social interaction stress on behavioural and physiological parameters in a stress-sensitive mouse strain. Behav Brain Res. 2011;216:576-84.

16. Powell ND, Sloan EK, Bailey MT, Arevalo JM, Miller GE, Chen E, Kobor MS, Reader BF, Sheridan JF, Cole SW. Social stress up-regulates inflammatory gene expression in the leukocyte transcriptome via beta-adrenergic induction of myelopoiesis. Proc Natl Acad Sci U S A. 2013;110:16574-9.

17. Wohleb ES, McKim DB, Shea DT, Powell ND, Tarr AJ, Sheridan JF, Godbout JP. Re-establishment of anxiety in stress-sensitized mice is caused by monocyte trafficking from the spleen to the brain. Biol Psychiatry. 2014;75:970-81.

18. Wohleb ES, Powell ND, Godbout JP, Sheridan JF. Stress-induced recruitment of bone marrow-derived monocytes to the brain promotes anxietylike behavior. J Neurosci. 2013:33:13820-33.

19. McKim DB, Weber MD, Niraula A, Sawicki CM, Liu X, Jarrett BL, RamirezChan K, Wang Y, Roeth RM, Sucaldito AD, et al. Microglial recruitment of IL-1 beta-producing monocytes to brain endothelium causes stressinduced anxiety. Mol Psychiatry. 2018;23:1421-31.

20. Lin JG, Chen WL. Acupuncture analgesia: a review of its mechanisms of actions. Am J Chin Med. 2008:36:635-45.

21. Zhao Z, Kim SC, Liu H, Zhang J, Wang Y, Cho IJ, Lee BH, Song CH, Lee CW, Yang $\mathrm{CH}$, et al. Manual acupuncture at PC6 ameliorates acute restraint stress-induced anxiety in rats by normalizing amygdaloid noradrenergic response. Evid Based Complement Alternat Med. 2017;2017:4351723.

22. Yi PL, Lu CY, Jou SB, Chang FC. Low-frequency electroacupuncture suppresses focal epilepsy and improves epilepsy-induced sleep disruptions. J Biomed Sci. 2015:22:49.

23. Moiraghi C, Poli P, Piscitelli A. An observational study on acupuncture for earthquake-related post-traumatic stress disorder: the experience of the Lombard Association of medical acupuncturists/acupuncture in the world, in Amatrice, Central Italy. Med Acupunct. 2019;31:116-22.

24. Hollifield M, Sinclair-Lian N, Warner TD, Hammerschlag R. Acupuncture for posttraumatic stress disorder: a randomized controlled pilot trial. J Nerv Ment Dis. 2007;195:504-13.

25. Kim YD, Heo I, Shin BC, Crawford C, Kang HW, Lim JH. Acupuncture for posttraumatic stress disorder: a systematic review of randomized controlled trials and prospective clinical trials. Evid Based Complement Alternat Med. 2013;2013:615857.

26. Grant S, Colaiaco B, Motala A, Shanman R, Sorbero M, Hempel S. Acupuncture for the treatment of adults with posttraumatic stress disorder: a systematic review and meta-analysis. J Trauma Dissociation. 2018;19:39-58.

27. Kwon CY, Lee B, Kim SH. Efficacy and underlying mechanism of acupuncture in the treatment of posttraumatic stress disorder: a systematic review of animal studies. J Clin Med. 2021;10:1575.
28. Fang YQ, Cai DJ, Zhou QZ, Yu SG, Peng XH, Zheng Z. Comparative research on intervention in anxiety behavior of PTSD-like rats by electroacupncture and repetitive transcranial magnetic stimulation and serum corticosterone. J Nanjing Univ TCM. 2012;28:357-9.

29. Li R: Electroacupuncture preconditioning ameliorates anxiety-like behavior and cognitive impairments in posttraumatic stress disorder model via upregulation of thioredoxin reductases. Master's Thesis, Shaanxi University of Chinese Medicine, Shaanxi, China 2016.

30. Ding N. Study of the effects on fear memory of PTSD rats intervened by calming shen-restoring consciousness-regulating kidney electroacupuncture therapy based on BDNF-TrkB-ERK pathway in Amygdala Master's Thesis. Chengdu: Chengdu University of Traditional Chinese Medicine; 2018. p. 2018.

31. Chen YHX, F Gu, TT, Wang SQ, Wang HN, Peng ZW. Effect of electroacupuncture pretreatment on anxiety-like behavior and expression of endogenous cannabinoid related genes in the prefrontal cortex of PTSD rats model. J Neurosci Ment Health. 2019, 19:658-662.

32. Zhou CH, Xue F, Xue SS, Sang HF, Liu L, Wang Y, Cai M, Zhang ZJ, Tan QR, Wang HN, Peng ZW. Electroacupuncture pretreatment ameliorates PTSD-like behaviors in rats by enhancing hippocampal neurogenesis via the Keap1/Nrf2 antioxidant signaling pathway. Front Cell Neurosci. 2019;13:275.

33. Wei KXH, CL, Chen XP, Cao H. Effects of electrical acupuncturing in Baihui acupoint on sleep phases of post-traumatic stress disorder rats and the mechanisms. Shaanxi J Tradit Chin Med. 2019, 40:1333-1335.

34. Zheng Y, He J, Guo L, Yao L, Zheng X, Yang Z, Xia Y, Wu X, Su Y, Xu N, Chen $Y$. Transcriptome analysis on maternal separation rats with depressionrelated manifestations ameliorated by electroacupuncture. Front Neurosci. 2019;13:314.

35. Wang WC, Lin TC, Kieber J, Tsai YC. Response regulators 9 and 10 negatively regulate salinity tolerance in rice. Plant Cell Physiol. 2019;60:2549-63.

36. Warde-Farley D, Donaldson SL, Comes O, Zuberi K, Badrawi R, Chao P, Franz M, Grouios C, Kazi F, Lopes CT, et al. The GeneMANIA prediction server: biological network integration for gene prioritization and predicting gene function. Nucleic Acids Res. 2010;38:W214-220.

37. Chen YH, Ivanic B, Chuang CM, Lu DY, Lin JG. Electroacupuncture reduces cocaine-induced seizures and mortality in mice. Evid Based Complement Alternat Med. 2013;2013:134610.

38. Yin CS, Jeong HS, Park HJ, Baik Y, Yoon MH, Choi CB, Koh HG. A proposed transpositional acupoint system in a mouse and rat model. Res Vet Sci. 2008:84:159-65.

39. Bourin M, Hascoet M. The mouse light/dark box test. Eur J Pharmacol. 2003:463:55-65.

40. Rodgers RJ, Dalvi A. Anxiety, defence and the elevated plus-maze. Neurosci Biobehav Rev. 1997;21:801-10.

41. Mucha M, Skrzypiec AE, Schiavon E, Attwood BK, Kucerova E, Pawlak R. Lipocalin-2 controls neuronal excitability and anxiety by regulating dendritic spine formation and maturation. Proc Natl Acad Sci USA. 2011;108:18436-41.

42. Lisowski P, Wieczorek M, Goscik J, Juszczak GR, Stankiewicz AM, Zwierzchowski L, Swiergiel AH. Effects of chronic stress on prefrontal cortex transcriptome in mice displaying different genetic backgrounds. J Mol Neurosci. 2013;50:33-57.

43. Stankiewicz AM, Goscik J, Majewska A, Swiergiel AH, Juszczak GR. The effect of acute and chronic social stress on the hippocampal transcriptome in mice. PLoS One. 2015;10:e0142195.

44. Czapski GA, Zhao Y, Lukiw WJ, Strosznajder JB. Acute systemic inflammatory response alters transcription profile of genes related to immune response and $\mathrm{Ca}(2+)$ homeostasis in hippocampus; relevance to neurodegenerative disorders. Int J Mol Sci. 2020;21:7838.

45. Peres DS, Theisen MC, Fialho MFP, Dalenogare DP, Rodrigues P, Kudsi SQ Bernardes LB, da Silva RNA, Lückemeyer DD, Sampaio TB, et al. TRPA1 involvement in depression- and anxiety-like behaviors in a progressive multiple sclerosis model in mice. Brain Res Bull. 2021;175:1-15.

46. Kim J, Suh YH, Chang KA. Interleukin-17 induced by cumulative mild stress promoted depression-like behaviors in young adult mice. Mol Brain. 2021;14:11.

47. Zhang ZJ, Wang XM, McAlonan GM. Neural acupuncture unit: a new concept for interpreting effects and mechanisms of acupuncture. Evid Based Complement Alternat Med. 2012;2012:429412. 
48. Lim S. WHO standard acupuncture point locations. Evid Based Complement Alternat Med. 2010;7:167-8.

49. Qiu T, Li L. Discussion on the Chinese edition of the WHO Standard Acupuncture Point Locations in the Western Pacific Region. Zhongguo Zhen Jiu. 2011; 31:827-830.

50. Khongrum J, Wattanathorn J. Laser acupuncture improves behavioral disorders and brain oxidative stress status in the valproic acid rat model of autism. J Acupunct Meridian Stud. 2015;8:183-91.

51. Davila-Hernandez A, Zamudio SR, Martinez-Mota L, Gonzalez-Gonzalez R, Ramirez-San Juan E. Antidepressant effects of acupoint stimulation and fluoxetine by increasing dendritic arborization and spine density in CA1 hippocampal neurons of socially isolated rats. Neurosci Lett. 2018;675:48-53.

52. Manni L, Aloe L, Fiore M. Changes in cognition induced by social isolation in the mouse are restored by electro-acupuncture. Physiol Behav. 2009;98:537-42.

53. Zhang HF, Li HX, Dai YC, Xu XJ, Han SP, Zhang R, Han JS. Electro-acupuncture improves the social interaction behavior of rats. Physiol Behav. 2015;151:485-93.

54. Xue F, Xue SS, Liu L, Sang HF, Ma QR, Tan QR, Wang HN, Zhou CH, Peng ZW. Early intervention with electroacupuncture prevents PTSD-like behaviors in rats through enhancing hippocampal endocannabinoid signaling. Prog Neuropsychopharmacol Biol Psychiatry. 2019;93:171-81.

55. Cheng CY, Lin JG, Tang NY, Kao ST, Hsieh CL. Electroacupuncture-like stimulation at the Baihui (GV20) and Dazhui (GV14) acupoints protects rats against subacute-phase cerebral ischemia-reperfusion injuries by reducing S100B-mediated neurotoxicity. PLoS One. 2014;9:e91426.

56. Cheng CY, Lin JG, Su SY, Tang NY, Kao ST, Hsieh CL. Electroacupuncturelike stimulation at Baihui and Dazhui acupoints exerts neuroprotective effects through activation of the brain-derived neurotrophic factor-mediated MEK1/2/ERK1/2/p90RSK/bad signaling pathway in mild transient focal cerebral ischemia in rats. BMC Complement Altern Med. 2014;14:92.

57. Yongxia R. Acupuncture treatment of Jacksonian epilepsy-a report of 98 cases. J Tradit Chin Med. 2006;26:177-8.

58. Shi YM, Wu M, Xu H. Progress on study and treatment of infantile enuresis. Zhongguo Zhong Xi Yi Jie He Za Zhi. 2003;23:792-4.

59. Lee $S$, Jha MK, Suk K. Lipocalin-2 in the inflammatory activation of brain astrocytes. Crit Rev Immunol. 2015;35:77-84.

60. Xing C, Wang X, Cheng C, Montaner J, Mandeville E, Leung W, van Leyen K, Lok J, Wang X, Lo EH. Neuronal production of lipocalin-2 as a help-me signal for glial activation. Stroke. 2014;45:2085-92.

61. Pinyopornpanish K, Chattipakorn N, Chattipakorn SC. Lipocalin-2: Its perspectives in brain pathology and possible roles in cognition. J Neuroendocrinol. 2019;31:e12779.

62. Mucha M, Skrzypiec AE, Schiavon E, Attwood BK, Kucerova E, Pawlak R. Lipocalin-2 controls neuronal excitability and anxiety by regulating dendritic spine formation and maturation. Proc Natl Acad Sci U S A. 2011;108:18436-41.

63. Ferreira AC, Da Mesquita S, Sousa JC, Correia-Neves M, Sousa N, Palha JA, Marques F. From the periphery to the brain: Lipocalin-2, a friend or foe? Prog Neurobiol. 2015;131:120-36.

64. Ferreira AC, Pinto V, Da Mesquita S, Novais A, Sousa JC, Correia-Neves M, Sousa N, Palha JA, Marques F. Lipocalin-2 is involved in emotional behaviors and cognitive function. Front Cell Neurosci. 2013;7:122.

65. Llorens F, Hermann P, Villar-Pique A, Diaz-Lucena D, Nagga K, Hansson O, Santana I, Schmitz M, Schmidt C, Varges D, et al. Cerebrospinal fluid lipocalin 2 as a novel biomarker for the differential diagnosis of vascular dementia. Nat Commun. 2020;11:619.

66. Sonninen TM, Hamalainen RH, Koskuvi M, Oksanen M, Shakirzyanova A, Wojciechowski S, Puttonen K, Naumenko N, Goldsteins G, Laham-Karam $\mathrm{N}$, et al. Metabolic alterations in Parkinson's disease astrocytes. Sci Rep. 2020;10:14474.

67. Gouweleeuw L, Naudé PJ, Rots M, DeJongste MJ, Eisel UL, Schoemaker $R G$. The role of neutrophil gelatinase associated lipocalin (NGAL) as biological constituent linking depression and cardiovascular disease. Brain Behav Immun. 2015:46:23-32.

68. Marijnissen RM, Naudé PJ, Comijs HC, Schoevers RA, Oude Voshaar RC. Waist circumference and neutrophil gelatinase-associated lipocalin in late-life depression. Brain Behav Immun. 2014;37:231-9.

69. Naudé PJ, den Boer JA, Comijs HC, Bosker FJ, Zuidersma M, Groenewold NA, De Deyn PP, Luiten PG, Eisel UL, Oude Voshaar RC. Sex-specific associations between Neutrophil Gelatinase-Associated Lipocalin (NGAL) and cognitive domains in late-life depression. Psychoneuroendocrinology. 2014;48:169-77.

70. Bannerman DM, Rawlins JN, McHugh SB, Deacon RM, Yee BK, Bast $\mathrm{T}$, Zhang WN, Pothuizen HH, Feldon J. Regional dissociations within the hippocampus-memory and anxiety. Neurosci Biobehav Rev. 2004;28:273-83.

71. Wang J, Barbas H. Specificity of primate amygdalar pathways to hippocampus. J Neurosci. 2018;38:10019-41.

72. Bagot RC, Parise EM, Peña CJ, Zhang HX, Maze I, Chaudhury D, Persaud B, Cachope R, Bolaños-Guzmán CA, Cheer JF, et al. Ventral hippocampal afferents to the nucleus accumbens regulate susceptibility to depression. Nat Commun. 2015;6:7062.

73. Schoenfeld TJ, Rhee D, Martin L, Smith JA, Sonti AN, Padmanaban V, Cameron HA. New neurons restore structural and behavioral abnormalities in a rat model of PTSD. Hippocampus. 2019;29:848-61.

74. File SE, Kenny PJ, Cheeta S. The role of the dorsal hippocampal serotonergic and cholinergic systems in the modulation of anxiety. Pharmacol Biochem Behav. 2000;66:65-72.

75. Dunn JD, Orr SE. Differential plasma corticosterone responses to hippocampal stimulation. Exp Brain Res. 1984;54:1-6.

76. McEwen BS, Milner TA. Understanding the broad influence of sex hormones and sex differences in the brain. J Neurosci Res. 2017;95:24-39.

77. Conrad CD. What is the functional significance of chronic stress-induced CA3 dendritic retraction within the hippocampus? Behav Cogn Neurosci Rev. 2006:5:41-60.

78. Woolley CS, Gould E, McEwen BS. Exposure to excess glucocorticoids alters dendritic morphology of adult hippocampal pyramidal neurons. Brain Res. 1990:531:225-31.

79. Anuncibay-Soto B, Pérez-Rodríguez D, Santos-Galdiano M, Font E, Regueiro-Purriños M, Fernández-López A. Post-ischemic salubrinal treatment results in a neuroprotective role in global cerebral ischemia. J Neurochem. 2016;138:295-306.

80. McEwen BS. The neurobiology of stress: from serendipity to clinical relevance. Brain Res. 2000;886:172-89.

81. Yao W, Zhang JC, Ishima T, Dong C, Yang C, Ren Q, Ma M, Han M, Wu $J$, Suganuma $\mathrm{H}$, et al. Role of Keap1-Nrf2 signaling in depression and dietary intake of glucoraphanin confers stress resilience in mice. Sci Rep. 2016;6:30659.

82. Shirayama Y, Yang C, Zhang JC, Ren Q, Yao W, Hashimoto K. Alterations in brain-derived neurotrophic factor (BDNF) and its precursor proBDNF in the brain regions of a learned helplessness rat model and the antidepressant effects of a TrkB agonist and antagonist. Eur Neuropsychopharmacol. 2015:25:2449-58

83. Hashimoto K. Essential role of Keap1-Nrf2 signaling in mood disorders: overview and future perspective. Front Pharmacol. 2018:9:1182.

84. Lai S, Wu G, Jiang Z. Glycyrrhizin treatment facilitates extinction of conditioned fear responses after a single prolonged stress exposure in rats. Cell Physiol Biochem. 2018;45:2529-39.

85. Joshi PC, Benerjee S. Effects of glucocorticoids in depression: role of astrocytes. AlMS Neurosci. 2018;5:200-10.

86. Otani N, Nawashiro H, Fukui S, Ooigawa H, Ohsumi A, Toyooka T, Shima K, Gomi H, Brenner M. Enhanced hippocampal neurodegeneration after traumatic or kainate excitotoxicity in GFAP-null mice. J Clin Neurosci. 2006;13:934-8.

87. Jahanshahi M, Sadeghi Y, Hosseini A, Naghdi N, Marjani A. The effect of spatial learning on the number of astrocytes in the CA3 subfield of the rat hippocampus. Singapore Med J. 2008;49:388-91.

88. Saavedra LM, Hernández-Velázquez MG, Madrigal S, Ochoa-Zarzosa A, Torner L. Long-term activation of hippocampal glial cells and altered emotional behavior in male and female adult rats after different neonatal stressors. Psychoneuroendocrinology. 2021;126:105164.

89. Lambert KG, Gerecke KM, Quadros PS, Doudera E, Jasnow AM, Kinsley CH. Activity-stress increases density of GFAP-immunoreactive astrocytes in the rat hippocampus. Stress. 2000;3:275-84.

90. Stephens MA, Wand G. Stress and the HPA axis: role of glucocorticoids in alcohol dependence. Alcohol Res. 2012;34:468-83.

91. de Kloet ER, Sutanto W, van den Berg DT, Carey MP, van Haarst AD, Hornsby CD, Meijer OC, Rots NY, Oitzl MS. Brain mineralocorticoid receptor diversity: functional implications. J Steroid Biochem Mol Biol. 1993;47:183-90. 
92. Harris AP, Holmes MC, de Kloet ER, Chapman KE, Seckl JR. Mineralocorticoid and glucocorticoid receptor balance in control of HPA axis and behaviour. Psychoneuroendocrinology. 2013;38:648-58.

93. Rush AJ, Trivedi MH, Wisniewski SR, Nierenberg AA, Stewart JW, Warden D, Niederehe G, Thase ME, Lavori PW, Lebowitz BD, et al. Acute and longer-term outcomes in depressed outpatients requiring one or several treatment steps: a STAR*D report. Am J Psychiatry. 2006;163:1905-17.

94. Duncan J, Johnson S, Ou XM. Monoamine oxidases in major depressive disorder and alcoholism. Drug Discov Ther. 2012;6:112-22.

95. Hung CF, Lung FW, Hung TH, Chong MY, Wu CK, Wen JK, Lin PY. Monoamine oxidase A gene polymorphism and suicide: an association study and meta-analysis. J Affect Disord. 2012;136:643-9.

96. Meyer JH, Wilson AA, Sagrati S, Miler L, Rusjan P, Bloomfield PM, Clark M, Sacher J, Voineskos AN, Houle S. Brain monoamine oxidase A binding in major depressive disorder: relationship to selective serotonin reuptake inhibitor treatment, recovery, and recurrence. Arch Gen Psychiatry. 2009;66:1304-12

97. Ishikawa R, Uchida C, Kitaoka S, Furuyashiki T, Kida S. Improvement of PTSD-like behavior by the forgetting effect of hippocampal neurogenesis enhancer memantine in a social defeat stress paradigm. Mol Brain. 2019;12:68

\section{Publisher's Note}

Springer Nature remains neutral with regard to jurisdictional claims in published maps and institutional affiliations.
Ready to submit your research? Choose BMC and benefit from:

- fast, convenient online submission

- thorough peer review by experienced researchers in your field

- rapid publication on acceptance

- support for research data, including large and complex data types

- gold Open Access which fosters wider collaboration and increased citations

- maximum visibility for your research: over $100 \mathrm{M}$ website views per year

At BMC, research is always in progress.

Learn more biomedcentral.com/submissions 